\title{
Chandra and XMM-Newton Observations of a Group of Galaxies HCG 62
}

\author{
Umeyo Morita, ${ }^{1}$ Yoshitaka Ishisaki,,${ }^{1}$ Noriko Y. Yamasaki, ${ }^{2}$ Naomi Ota,${ }^{3}$ \\ Naomi Kawano, ${ }^{4}$ Yasushi Fukazawa, ${ }^{4}$ and Takaya Ohashi ${ }^{1}$ \\ ${ }^{1}$ Department of Physics, Tokyo Metropolitan University, 1-1 Minami-Osawa, Hachioji, Tokyo 192-0397 \\ umeyo@phys.metro-u.ac.jp \\ ${ }^{2}$ Institute of Space and Astronautical Science (ISAS/JAXA), \\ 3-1-1 Yoshinodai, Sagamihara, Kanagawa 229-8510 \\ ${ }^{3}$ Cosmic Radiation Laboratory, RIKEN, 2-1 Hirosawa, Wako, Saitama 351-0198 \\ ${ }^{4}$ Department of Physical Science, School of Science, Hiroshima University, \\ 1-3-1 Kagamiyama, Higashi-Hiroshima, Hiroshima 739-8526
}

(Received 2005 0; accepted 2005 0)

\begin{abstract}
We present results from Chandra and XMM-Newton observations of the bright group of galaxies HCG 62. There are two cavities at about $30^{\prime \prime}$ northeast and $20^{\prime \prime}$ southwest of the central galaxy in the Chandra image. The energy spectrum shows no significant change in the cavity compared with that in the surrounding region. The radial X-ray profile is described by a sum of $3-\beta$ components with core radii about 2, 10, and $160 \mathrm{kpc}$, respectively. We studied radial distributions of temperature and metal abundance with joint spectral fit for the Chandra and XMM-Newton data, and two temperatures were required in the inner $r<2^{\prime}$ (35 kpc) region. The sharp drop of temperature at $r \sim 5^{\prime}$ implies the gravitational mass density even lower than the gas density, suggesting the gas may not be in hydrostatic equilibrium. Fe and $\mathrm{Si}$ abundances are 1-2 solar at the center and drop to about 0.1 solar at $r \sim 10^{\prime}$. O abundance is less than 0.5 solar and shows a flatter profile. Observed metal distribution supports the view that iron and silicon are produced by type Ia supernova in the central galaxy, while galactic winds by type II supernova have caused wide distribution of oxygen. The supporting mechanism of the cavity is discussed. Pressure for the sum of electrons and magnetic field is too low to displace the hot group gas, and the required pressure due to high energy protons are nearly 700 times higher than the electron pressure. This leaves the origin of the cavities a puzzle, and we discuss other possible origins of the cavities.

Key words: galaxies: clusters: individual (HCG 62) — galaxies: abundances — galaxies: ISM — X-rays: galaxies — X-rays: ISM, cavity
\end{abstract}




\section{Introduction}

Groups of galaxies hold significantly less amount of hot gas compared with rich clusters on the average, and the apparent deficiency of baryons in these low-mass systems is a problem in explaining the baryon budget in rich systems in terms of hierarchical merging scenario (Voit 2005). The observations of compact groups of galaxies are important to search for the hidden form of baryons and their release mechanism, which would be strongly connected with dynamics of gas and galaxies. In particular, some groups dominated by bright central galaxies have shown gas features which are strongly affected by the activity of the central galaxies. High sensitivity X-ray observations of groups of galaxies are powerful method to look into the role of central galaxies in terms of metal distribution and gas morphologies.

The striking gas features most likely caused by the central galaxies are the X-ray cavities. The cavities are circular regions showing a significant depression of X-ray surface brightness. Nearly 20 cavities have been recognized in clusters and groups with high resolution images taken by ROSAT and Chandra (Bîrzan et al. 2004, hereafter B04; Dunn \& Fabian 2004; Dunn et al. 2005, hereafter D05). They are located typically at 10-30 kpc from the central galaxies, and strong correlation with radio lobes are seen in about 10 systems. Remarkable cases are seen in the Perseus (e.g., Boehringer et al. 1993; Fabian et al. 2000) and Hydra A (e.g. McNamara et al. 2000) clusters, both showing strong correlation with the $1.4 \mathrm{GHz}$ radio lobes. Several giant elliptical galaxies with radio robes, e.g., M84 (Finoguenov \& Jones 2001), NGC 4636 (Ohto

at al. 2003), are also known as nesting X-ray cavities. The remaining half of the cavities, on the other hand, are not associated with radio lobes, and they are designated as ghost cavities. The one in A 2597 (McNamara et al. 2001) or the outer depressions in Perseus (Fabian et al. 2000) are examples. Cavities are thought to be produced by jets or buoyant bubbles which are directly connected with the activity of central radio galaxies.

A subsonic displacement of the gas would create a low density, rising bubble keeping the pressure balance with the surrounding ICM. It appears to be in general supposed for cavities that non-thermal pressure originated in relativistic particles and/or magnetic fields in the radio lobe is probably large enough to balance with the surrounding ICM gas pressure (Fabian et al. 2002). This pseudo-pressure balance is justified by the fact that there are no evidence for shock-heated gas around the radio lobes in almost all of the X-ray cavities observed so far, except for MKW 3s (Mazzotta et al. 2002). This general scenario has been modeled theoretically, and has at least qualitatively reproduced the morphology of cavities (e.g., Churazov et al. 2001). Energy density of relativistic electrons inferred from the synchrotron radio emission is almost always smaller than that required to offset the hot gas by orders of magnitude, and it is discussed that energy density of protons are higher than those due to electrons by factors of 100-1000 (D05). However, there is no direct evidence indicating that such a high energy density is really carried by protons. This situation is the severest in the case of ghost cavities. In this view, it 
is important to examine ghost cavities in groups of galaxies where the gas is relatively cool and non-thermal effect can be recognized somewhat easily.

Detailed studies on the metal distribution in clusters and groups have been carried out using ASCA, BeppoSAX, Chandra and XMM-Newton. Distribution of iron and silicon indicate strong central concentration in clusters and groups characterized by bright central galaxies, and the excess iron mass is found to correlate with the luminosity of the cD galaxy (De Grandi et al. 2004). This indicates that iron and silicon (main products from type Ia supernova; SN Ia) trace the enhanced star-formation activity in bright galaxies. On the other hand, distribution of oxygen (i.e. type II supernova product; SN II) is not well understood. Matsushita et al. (2003) showed that oxygen distribution around M 87 is flatter than those of iron and silicon, with the level about half as much as the others. Such low oxygen abundances are also derived in the centers of other clusters and groups (e.g. Buote et al. 2003b; Xue et al. 2004). For the study of oxygen distribution, low temperature systems such as groups of galaxies are suitable targets.

In this paper, we report the results from Chandra and XMM-Newton observations of HCG 62, which is one of the nearest Hickson compact galaxy groups (Hickson et al. 1989). The whole group consists of 63 galaxies (Mulchaey et al. 2003) within a radius of 50' (900 kpc), but the central region is dominated by 4 galaxies. HCG 62 is the brightest group of galaxies in the X-ray band, and the extended X-ray emission was first discovered by Ponman \& Bertram (1993) from the ROSAT PSPC observation. Based on the ASCA observation, Fukazawa et al. (2001) detected excess hard X-ray emission, and Finoguenov \& Ponman (1999) report strong central concentration of iron. Using the high resolution image of Chandra, Vrtilek et al. (2001,2002) detected two ghost cavities, which is the first report of cavities in groups of galaxies.

This paper is organized as follows: In $\S 2$ we describe the Chandra and XMM-Newton observations and the data reduction. In $\S 3$ we give the image of HCG 62 of both Chandra and XMM-Newton, in $\S 4$ we describe the X-ray cavity structure using Chandra image. In $\S 5$ we present our results on the temperature profiles and the abundances profiles of $\mathrm{Fe}$ and $\alpha$-elements ( $\mathrm{Si}, \mathrm{Mg}$, and $\mathrm{O}$ ). In $\S 6-8$ we give discussions of the obtained results, and finally we summarize our conclusions in $\S 9$. Throughout this paper we adopt $\Omega_{\Lambda}=1-\Omega_{\mathrm{M}}=0.73$ and $h_{70} \equiv H_{0} /\left(70 \mathrm{~km} \mathrm{~s}^{-1} \mathrm{Mpc}^{-1}\right)=1 ; 1^{\prime}$ corresponds to $17.8 \mathrm{kpc}$ at $z=0.0145$. The quoted errors indicate the $90 \%$ confidence range, unless otherwise stated. We use the solar abundance ratio of Anders and Grevesse (1989).

\section{Observation and Data Reduction}

\subsection{Chandra Observation}

HCG 62 was observed on 25 January 2000 with the Advanced CCD imaging Spectrometer (ACIS) I2, I3, S2, S3, and S4 chips operated at the CCD temperature of $-110^{\circ} \mathrm{C}$ with a frame readout time of $3.24 \mathrm{~s}$. We used the data of only the ACIS-S3 chip $\left(8.4^{\prime} \times 8.4^{\prime}\right)$ covering the cen- 
tral part of HCG 62 in this paper. The pointing coordinates were $\left(12^{\mathrm{h}} 59^{\mathrm{m}} 05^{\mathrm{s}} 70,-09^{\circ} 12^{\prime} 20 !^{\prime \prime} 00\right)$ (J2000) and the total exposure was $49.15 \mathrm{ks}$. According to the "Chandra Aspect" web page, http://cxc.harvard.edu/cal/ASPECT/, the astrometry offset of RA $=-0.03^{\prime \prime}$, Dec $=0.22^{\prime \prime}$ has been corrected for in the data, providing a radius of 0.6 arcsec as the absolute position accuracy in $90 \%$ confidence. The CCD temperature of ACIS was reduced to $-120^{\circ} \mathrm{C}$ soon after the observation of HCG 62 in January 2000 due to the increase of charge transfer inefficiency (CTI) caused by the radiation damage in orbit (Grant et al. 2005). The data were telemetered in the Faint mode, and events with the ASCA grades of 0, 2, 3, 4, and 6 were used. Bad pixels, bad columns, and the columns next to bad columns and to the chip node boundaries are excluded. In order to remove periods of anomalous background levels, we further filtered the events using the $0.3-10 \mathrm{keV}$ band light curve of the whole ACIS-S3 chip in $200 \mathrm{~s}$ bin, and discarded periods which exceeded by $3 \sigma$ above the mean quiescent rate of $4.5 \mathrm{c} / \mathrm{s} / \mathrm{chip}$. The net exposure time after the screening was 48,013 s. The data reduction was performed using CIAO version 3.1 with CALDB version 2.29, and the spectral fitting was done by XSPEC version 11.3.0t.

All of the X-ray spectra were extracted using the pulse-height invariant (PI) values, which were recomputed using the latest gain file acisD1999-09-16gainN0005.fits, appropriate for the $\mathrm{CCD}$ temperature of $-110^{\circ} \mathrm{C}$. In the spectral fitting, we initially generated the response matrix file (RMF) of the ACIS-S3 using the CIAO "mkrmf" task with the input FEF (FITS Embedded Function) file of acisD1999-09-16fef_phaN0002.fits, which was chosen by default. However, we found that the Si line of the IGM emission was significantly broader than the response $\left(\sigma=42_{-8}^{+9} \mathrm{eV}\right)$, which caused the fit statistics not acceptable. In order to examine whether this broad Si line is a target specific issue or a common calibration problem, we further checked the neutral Si line, $\mathrm{K}_{\alpha 1}=1739.98 \mathrm{eV}$ and $\mathrm{K}_{\alpha 2}=1739.38 \mathrm{eV}$, which is originated in the instrumental background of the CCD, using the blank-sky data obtained at $-110^{\circ} \mathrm{C}$ (figure 1 (a)). The fit result gave the Gaussian $\sigma=29 \pm 4 \mathrm{eV}(\mathrm{FWHM}=68 \pm 9 \mathrm{eV})$ and the line center energy of $1753 \pm 3 \mathrm{eV}$, which is significantly broad, too. We splitted the blank-sky spectrum into two, dividing the integration region into half, i.e. upper rows and lower rows of the CCD, although the results were similar. This result indicates that the charge transfer inefficiency (CTI) correction is working well.

We therefore concluded that the broad Si line was resulted because the generated RMF had too sharp Gaussian core. In fact, the derived Si line width for the blank-sky is consistent with a calibration document, ACIS Memo \#182 by LaMarr (2000). The FWHM of the Gaussian core for the RMF denoted in the FEF file is G1_FWHM $=73 \mathrm{eV}$ for ACIS-S3 (CCD_ID=7) at the energy of $1.8 \mathrm{keV}$, while it should be around $125 \mathrm{eV}$ according to the memo. Our blank-sky data indicated $\sqrt{73^{2}+68^{2}}=100 \mathrm{eV}$, which is slightly better than the value in the LaMarr's memo, possibly due to the updated gain file. We therefore created a new FEF file to adopt broader Gaussian core over the whole energy range, modifying the G1_FWHM column into $(16 \times$ ENERGY + G1_FWHM). Using the new FEF file, we generated the RMF files with the 
"mkrmf" task for the following analysis.

Regarding the "blank-sky" background, we adopted a series of observations compiled by Markevitch (http://cxc.harvard.edu/contrib/maxim/acisbg/), when the CCD temperature was $-110^{\circ} \mathrm{C}$. The "blank-sky" background events were also screened to remove background flares in the same way as described above. The additional component due to the Galactic soft background are considered in $\S 2.3$.

\subsection{XMM-Newton observation}

The XMM-Newton observation of HCG 62 was carried out on 15 January 2003, assigned for $12.6 \mathrm{ks}$. The EPIC cameras were operated in full-frame mode for MOS, and in extendedfull-frame mode for pn. The medium filters were used for both cameras. XMM-Newton covers the wider field of view of $r \lesssim 15^{\prime}$ than Chandra, while the angular resolution of $15^{\prime \prime}$ half-power diameter is broader. Data reduction was performed using SAS version 6.0, and the spectral fitting was done by XSPEC version 11.3.0t. We selected events with pattern 0-12 for MOS and 0-4 for pn, and flag $=0$ for both. Bad pixels and bad columns were excluded. We calculated the count rate distribution with $100 \mathrm{~s}$ intervals over the $0.3-10 \mathrm{keV}$ range using the whole chip of each sensor, and rejected periods by requiring all the count rates to be within $\pm 2 \sigma$ around the mean. We iterated the process until the number of rejected data in a step reached less than $5 \%$ of the $2 \sigma$ compared with the previous value. Because we did not find large time variation nor flares, the net exposure time after the screening was 12.6, 12.5, and $9.2 \mathrm{ks}$ for MOS1, MOS2, and pn, respectively.

To correct for the vignetting effect, the SAS "evigweight" task was applied for each event file. All the X-ray spectra were extracted using the vignetting-weighted events. The response files for spectral fittings were generated in the standard way with the SAS "rmfgen" and "arfgen" tasks at on-axes of the X-ray mirrors. The MOS1 and MOS2 spectra are summed up, and the energy range of $1.4367-1.5367 \mathrm{keV}$, where the background $\mathrm{Al}-\mathrm{K}_{\alpha}(1.4867 \mathrm{keV})$ is strong as seen in figure 1 (b) and (c), is ignored in the spectral fitting for both MOS and pn.

The background event dataset created by Read \& Ponman (2003) was adopted as the "blank-sky" background for the XMM-Newton observation. We applied the same selection criteria as the source events to the "blank-sky" background. The "blank-sky" background was scaled to 0.94 for MOS1, 1.00 for MOS2, and 0.84 for pn, respectively. These factors were determined by the count rate ratio within $r<14^{\prime}$ from the central galaxy HCG 62a in 10$12 \mathrm{keV}$ (MOS) or 12-14 keV (pn) band, where the instrumental background is dominant for each sensor. Katayama et al. (2004) and Nevalainen et al. (2005) have studied the background data of XMM-Newton in detail, and it is reported that the the $90 \%$ confidence background uncertainty is $\pm 5 \%$ in $4-7 \mathrm{keV}$ and $\pm 20 \%$ in $0.8-1 \mathrm{keV}$. It is confirmed that our results does not change significantly within errors when this level of uncertainty is considered. 

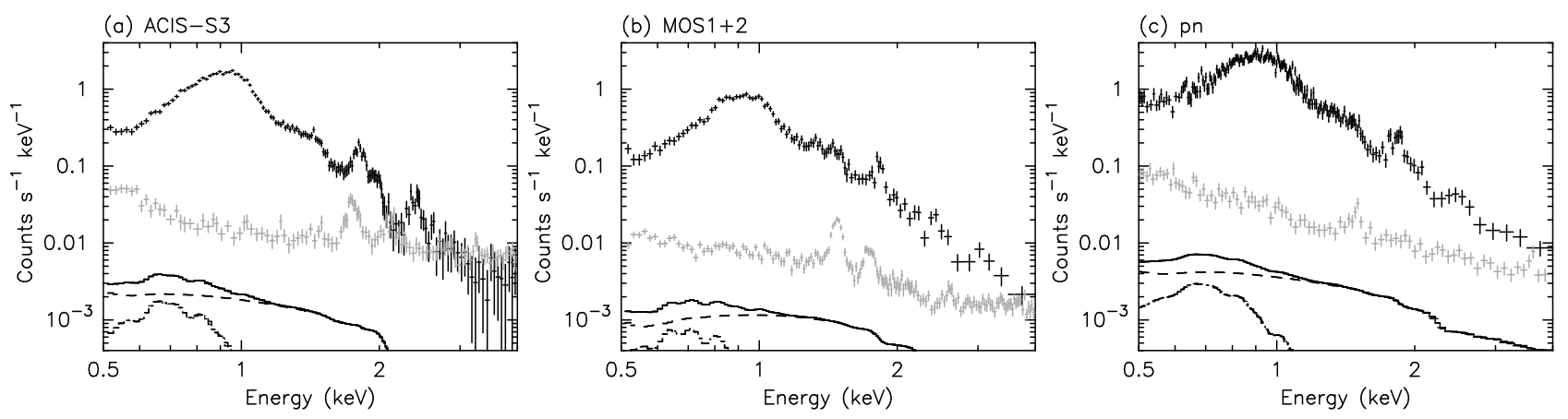

Fig. 1. X-ray spectra of (a) Chandra ACIS-S3, (b) XMM-Newton MOS1+2, and (c) pn, in 0.5-4 keV within $r<2^{\prime}$ from the central galaxy HCG 62a, subtracted by the "blank-sky" background. The "blank-sky" spectra are indicated by gray lines. The additional background components of the $0.3 \mathrm{keV}$ Mekal and the $\Gamma=1.5$ power-law models are shown by dashed black lines, and their sum is indicated by solid black lines.

\subsection{Background estimation}

HCG 62 is located near the edge of the North Polar Spur (NPS), which is a large soft $\mathrm{X}$-ray Galactic structure. The ROSAT All-Sky Survey (RASS) image at $3 / 4 \mathrm{keV}$ band in this region indicates a soft X-ray excess around the position of HCG 62, although significant fraction of the excess is probably caused by HCG 62 itself. This emission looks more extended than the Chandra and XMM-Newton fields of view in the previous observations with ROSAT and ASCA. Therefore, we cannot use the events in outer region as the background, and we need to estimate the influence of the soft X-ray background to our HCG 62 data using the "blank-sky" background.

There are four fields near HCG 62 already observed with Chandra, Q1246-0542, NGC 4697, NGC 4594, and NGC 4782. We found that the intensities below $2 \mathrm{keV}$ in source-free regions in these fields are higher than the level of the "blank-sky" background, while the hard band intensities show no excess. By fitting the energy spectra of the soft excess component with the XSPEC Mekal model, we obtained the temperature to be $\sim 0.3 \mathrm{keV}$, consistent with the previous result for the Galactic soft emission from NPS (Inoue et al. 1980). The surface brightness in the four nearby fields differ significantly, ranging in (5-10) $\times 10^{-10}$ photons cm ${ }^{-2} \mathrm{~s}^{-1} \operatorname{arcsec}^{-2}$ in $0.5-1 \mathrm{keV}$ band, and we need to estimate the soft X-ray background component based on the data of the HCG 62 field.

We examined the radial profile at $r<15^{\prime}$ of the XMM-Newton data in $0.5-1 \mathrm{keV}$ band by fitting with a sum of a double $\beta$ model and a constant intensity representing the soft X-ray background. In fitting the profile, parameters for the double $\beta$ component were fixed at the ROSAT values (Zabludoff \& Mulchaey 2000), and only the level of the constant component was varied as a free parameter. The resultant surface brightness is $2 \times 10^{-11}$ photons $\mathrm{cm}^{-2} \mathrm{~s}^{-1} \operatorname{arcsec}^{-2}$ in $0.5-1 \mathrm{keV}$ band. In the following analysis, the excess soft component is assumed to be a thermal emission with the temperature of $0.3 \mathrm{keV}$ and abundance of 1 solar, with the normal- 
ization fixed at the level of the radial profile fit above. We do not apply interstellar absorption for this component, since its origin is supposed to be a nearby region in our galaxy. When the normalization of the $0.3 \mathrm{keV}$ component is varied in the spectral fit, it agrees with the level derived from the radial profile, within the $90 \%$ confidence limit for both Chandra and XMM-Newton. In fact, the estimated additional soft component is much fainter than those in the four nearby fields, even less than the "blank-sky" background component. The "blank-sky" background also contains a certain fraction of the Galactic soft emission, and the HCG 62 field appears to require only the same level of soft X-ray background. We have also confirmed that our result did not change significantly within errors even doubling the intensity of the estimated soft background component.

As for the additional hard X-ray background component, we take into account the excess emission detected with ASCA (Fukazawa et al. 2001). In the 2-10 keV range, the observed flux of the $\Gamma=1.5$ power-law component with ASCA is $1.0 \times 10^{-12} \mathrm{ergs} \mathrm{cm}^{-2} \mathrm{~s}^{-1}$ at the ring-like area with inner and outer radii to be $5^{\prime}$ and $15^{\prime}$. We assumed the same power-law spectrum in our analysis, with the spatial distribution uniform over the whole HCG 62 field for both Chandra and XMM-Newton.

In the following analysis, the soft and the hard components were added as additional spectral models with fixed parameters. We however confirmed that the results did not vary significantly at the $90 \%$ confidence level, even if we did not apply these backgrounds. In figure 1 , the soft and the hard X-ray background components as well as the "blank sky" background are compared with the IGM spectrum for each instrument.

\section{X-ray Image}

\subsection{Chandra image}

The Chandra image of HCG 62 taken with ACIS-S3 in the $0.5-4 \mathrm{keV}$ energy band is shown in figure 2 (a). We corrected for background and exposure, and applied Gaussian smoothing. The positional dependence of the telescope and the detector responses were corrected with an exposure map. The IGM emission is clearly observed, and it is extended around the central galaxy HCG 62a. The brightest region has a radius of about $1^{\prime}$ from HCG $62 \mathrm{a}$.

We searched for discrete X-ray sources in the ACIS-S3 field using the CIAO "wavdetect" task. Choosing a significance parameter of $10^{-6}$ for images in the energy bands $0.5-10 \mathrm{keV}$, 0.5-2.0 keV, and 2.0-10.0 keV, we detected 50 sources in total, including HCG 62a, 62b, 62c, and 62-66. We have detected all the point sources previously identified in the SEXSI catalog (Harrison et al. 2003) using the same Chandra data. Optical properties of the member galaxies within the Chandra ACIS-S3 field of view are summarized in table 1, and the optical image is shown in figure 2 (b). The X-ray detected positions and the ACIS-S3 count are summarized in table 2. The X-ray position of HCG $62 \mathrm{a}$ and HCG $62 \mathrm{~b}$ well agree with the optical coordinate 
Table 1. Optical properties of HCG 62 and member galaxies.

\begin{tabular}{|c|c|c|c|c|c|c|}
\hline \multirow[t]{2}{*}{ Object } & \multicolumn{2}{|c|}{ Optical coords. $(\mathrm{J} 2000)^{*}$} & \multirow[t]{2}{*}{$z^{*}$} & \multirow{2}{*}{$\begin{array}{c}\text { Diameter }{ }^{\dagger} \\
(\operatorname{arcsec})\end{array}$} & \multirow{2}{*}{$\begin{array}{c}B^{\dagger} \\
(\mathrm{mag})\end{array}$} & \multirow[t]{2}{*}{ Type ${ }^{\ddagger}$} \\
\hline & R.A. & Dec. & & & & \\
\hline HCG $62 \ldots \ldots \ldots \ldots \ldots \ldots \ldots$ & $12^{\mathrm{h}} 53^{\mathrm{m}} 06^{\mathrm{s}} 1$ & $-09^{\circ} 12^{\prime} 16^{\prime \prime} 3$ & 0.0145 & - & - & Group \\
\hline HCG $62 \mathrm{a}$ & $12^{\mathrm{h}} 53^{\mathrm{m}} 05.6$ & $-09^{\circ} 12^{\prime} 13^{\prime \prime}$ & 0.0143 & 59.9 & 13.79 & E3 / S0 \\
\hline HCG $62 \mathrm{~b}$ & $12^{\mathrm{h}} 53^{\mathrm{m}} 04^{\mathrm{s}} 4$ & $-09^{\circ} 11^{\prime} 59^{\prime \prime}$ & 0.0119 & 42.9 & 14.21 & $\mathrm{~S} 0 / \mathrm{S} 0$ \\
\hline HCG $62 \mathrm{c}$ & $12^{\mathrm{h}} 53^{\mathrm{m}} 09.7$ & $-09^{\circ} 11^{\prime} 51^{\prime \prime}$ & 0.0148 & 40.2 & 15.00 & $\mathrm{~S} 0 / \mathrm{E}$ \\
\hline HCG $62 \mathrm{~d} \ldots$ & $12^{\mathrm{h}} 53^{\mathrm{m}} 06 \mathrm{~s} 6$ & $-09^{\circ} 15^{\prime} 26^{\prime \prime}$ & 0.0136 & 19.7 & 16.30 & $\mathrm{E} 2 /-$ \\
\hline HCG $62-66^{\S}$. & $12^{\mathrm{h}} 52^{\mathrm{m}} 48.7$ & $-09^{\circ} 13^{\prime} 22^{\prime \prime}$ & 0.0161 & - & - & $-1-$ \\
\hline
\end{tabular}

* Optical coordinates and redshift $z$ by Mulchaey et al. (2003) for HCG 62 and by Zabludoff \& Mulchaey (2000) for member galaxies.

$\dagger B$ band effective diameter, $D_{B}=\sqrt{A_{B} / \pi}$, and magnitude within $\mu_{B}=24.5 \mathrm{mag} \operatorname{arcsec}^{-2}$ isophote by Hickson et al. (1989).

¥ Hubble morphological type classification by Hickson et al. (1989) or Shimada et al. (2000).

$\S$ Because HCG 62-66 is not catalogued by Hickson et al. (1989), Diameter, B, and Type are left blank.

Table 2. X-ray properties of detected galaxies by Chandra ACIS-S3.

\begin{tabular}{|c|c|c|c|c|c|}
\hline \multirow[t]{2}{*}{ Object } & \multicolumn{2}{|c|}{ X-ray position $(\mathrm{J} 2000)^{*}$} & \multirow{2}{*}{$\begin{array}{l}\text { Pos. diff. }{ }^{\dagger} \\
(\operatorname{arcsec})\end{array}$} & \multirow{2}{*}{$\begin{array}{c}\text { Obs. count } \ddagger \\
\text { (cts) }\end{array}$} & \multirow{2}{*}{$\begin{array}{c}\text { Extended } \\
\mathrm{Y} / \mathrm{N}\end{array}$} \\
\hline & R.A. & Dec. & & & \\
\hline HCG $62 \mathrm{a}$ & $12^{\mathrm{h}} 53^{\mathrm{m}} 05.63$ & $-09^{\circ} 12^{\prime} 13^{\prime \prime} 7$ & 0.8 & $4158 \pm 65$ & Y \\
\hline HCG $62 \mathrm{~b}$ & $12^{\mathrm{h}} 53^{\mathrm{m}} 04.43$ & $-09^{\circ} 11^{\prime} 59^{\prime \prime} 4$ & 0.6 & $1344 \pm 37$ & Y \\
\hline $\mathrm{HCG} 62 \mathrm{c}$ & $12^{\mathrm{h}} 53^{\mathrm{m}} 09.76$ & $-09^{\circ} 11^{\prime} 55^{\prime \prime} 7$ & 4.8 & $331 \pm 19$ & Y ? \\
\hline HCG $62 d^{\S}$ & - & - & - & $36 \pm 7$ & Y? \\
\hline HCG $62-66$ & $12^{\mathrm{h}} 52^{\mathrm{m}} 48.57$ & $-09^{\circ} 13^{\prime} 25^{\prime \prime} 7$ & 4.2 & $30 \pm 7$ & Y ? \\
\hline
\end{tabular}

* Detected position by the CIAO "wavdetect" task, with $90 \%$ confidence position accuracy of 0.6 " radius.

$\dagger$ Positional difference between X-ray and optical.

$\ddagger$ Observed count within a radius of $10^{\prime \prime}$ in $0.5-4 \mathrm{keV}$ including IGM emission but subtracted by the "blank-sky".

$\S$ HCG 62 d was not detected by the "wavdetect" task, so that the optical position was utilized. 

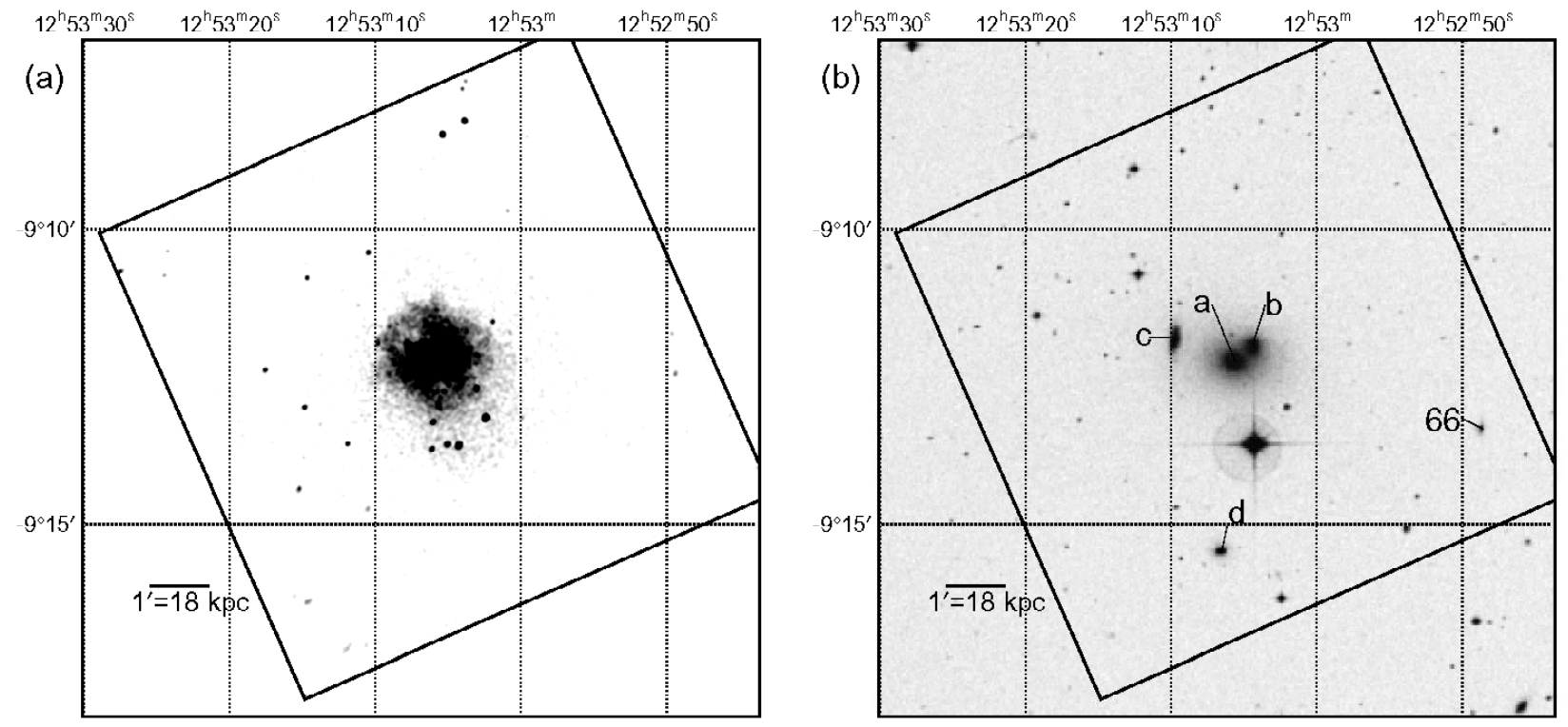

Fig. 2. (a) Gaussian smoothed Chandra ACIS-S3 X-ray image in the $0.5-4 \mathrm{keV}$ band. The smoothing scale is $\sigma=1.5^{\prime \prime}$ and the image is corrected for exposure and background. (b) STScI Digitized Sky Survey (DSS) image of HCG 62. The ACIS-S3 field is indicated by a square, and the member galaxies are denoted by a, b, c, d, and 66. Coordinates of images are J2000.

given in Zabludoff \& Mulchaey (2000) within $<1$ ". However, HCG 62c and HCG 62-66 indicate different positions by about $5^{\prime \prime}$ and $4^{\prime \prime}$. This is due probably to the extent of the galaxies and/or to the gas stripping, because the probability of miss-identification of background or foreground object within $5^{\prime \prime}$ radius is calculated to be only $1.5 \%$.

The X-ray emission of HCG 62a is dominated by the extended gas, for which we will describe the properties later. The HCG $62 \mathrm{~b}$ galaxy was also found to be significantly more extended than the point spread function of Chandra. We have fitted the radial brightness profile, $S(r)$, of HCG $62 \mathrm{~b}$ with a single $\beta$ model,

$$
S(r)=S_{0}\left[1+\left(r / R_{\mathrm{c}}\right)^{2}\right]^{-3 \beta+1 / 2},
$$

and obtained a core radius, $R_{\mathrm{c}}=1.5_{-0.6}^{+1.1}$ arcsec, $\beta=0.5_{-0.1}^{+0.2}$, and the normalization, $S_{0}=0.8_{-0.3}^{+0.5} \times$ $10^{-6}$ photons $\mathrm{cm}^{-2} \mathrm{~s}^{-1}$ arcsec ${ }^{-2}$. There is also an indication for the HCG 62c galaxy to be extended in the Chandra image in the direction of the optical major axis (north - south), although it is not clear whether it is due to the extended IGM emission because of the poor statistics of HCG 62c. The HCG 62d galaxy was not detected by the "wavdetect" task, however, the "blank-sky" subtracted count exhibited a clear excess when integrated within 10" around the optical position as shown in table 2. It is supposed that the extended feature of HCG $62 \mathrm{~d}$ may have hampered the detection by "wavdetect".

In the following analysis, the detected sources were excluded except for HCG 62a and HCG 62 d. Since HCG $62 \mathrm{a}$ and HCG $62 \mathrm{~b}$ are closely located, we need to separate the HCG $62 \mathrm{~b}$ 

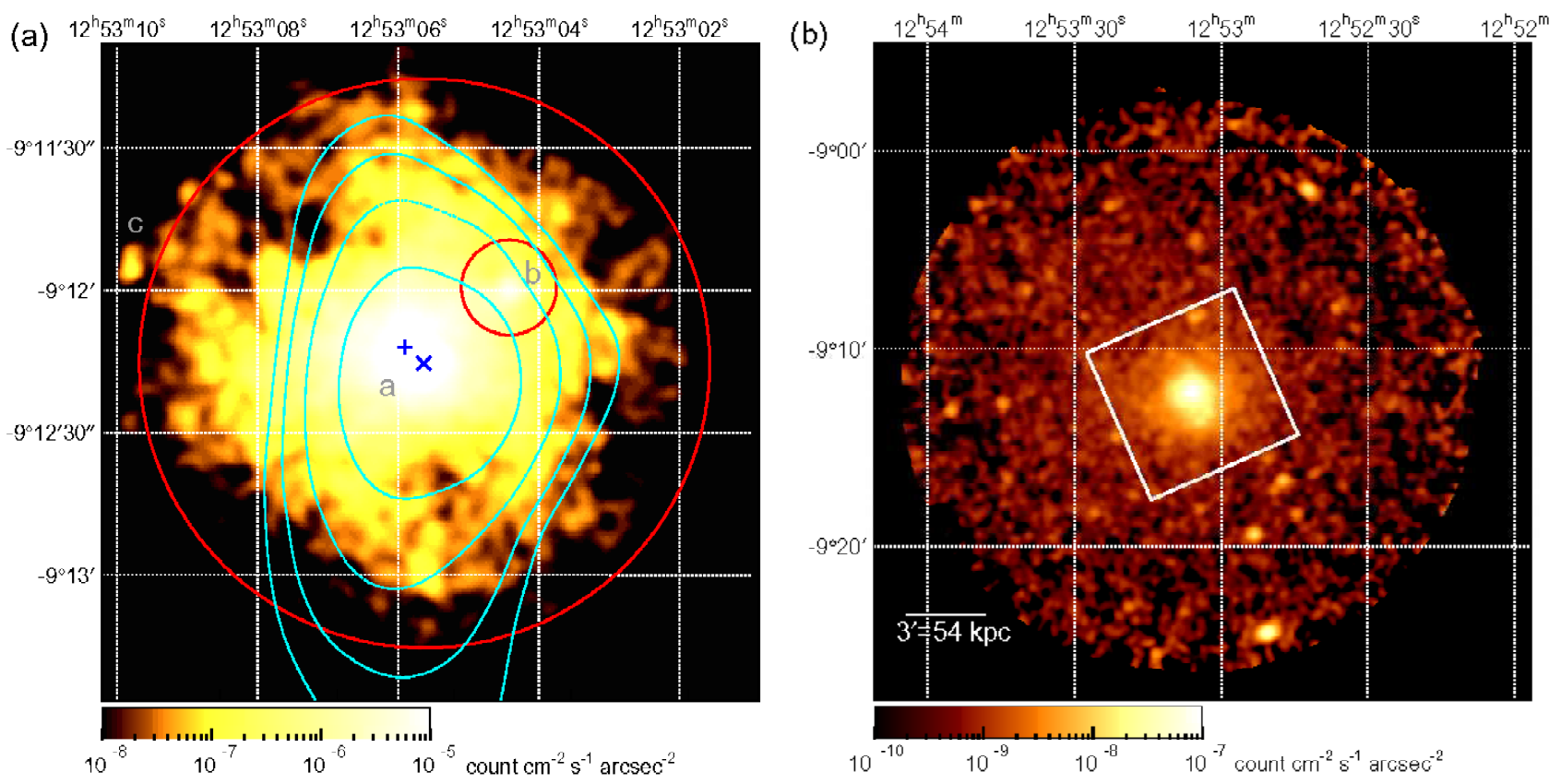

Fig. 3. (a) Same as figure 2, but the central region of HCG 62 is expanded. The detected point sources other than HCG $62 \mathrm{a}, 62 \mathrm{~b}$ and $62 \mathrm{c}$ are excluded. The central positions for the 2 -dimensional 2 - $\beta$ fit are denoted by cross and plus corresponding to the narrower and the wider $\beta$ components. The red circles indicate a radius of $1^{\prime}$ from HCG $62 \mathrm{a}$ and $10^{\prime \prime}$ from HCG $62 \mathrm{~b}$, respectively. The overlaid contour represent a radio intensity map at $1.4 \mathrm{GHz}$ with $45^{\prime \prime}$ FWHM resolution, derived from the NRAO VLA Sky Survey (NVSS; Condon et al. 1998). (b) Combined image with MOS1 and MOS2 detectors in the 0.5-4 keV band (J2000). The image is Gaussian smoothed with $\sigma=10^{\prime \prime}$ and corrected for exposure and background. A white square represents the ACIS-S3 field.

component in order to examine the IGM structure. It is notable that the redshift of HCG $62 \mathrm{~b}$ is smaller than the group redshift of $z=0.0145$ by $0.0026(\sim 11 \mathrm{Mpc})$, hence HCG $62 \mathrm{~b}$ is probably not interacting with the IGM around the group core. We produced two radial profiles in $0.5-4 \mathrm{keV}$ band centered on HCG $62 \mathrm{a}$ and HCG $62 \mathrm{~b}$, and fitted them with a double $\beta$ (HCG 62a) and a single $\beta$ (HCG 62b) models, respectively. With these fits, we evaluated the relative intensity of HCG $62 \mathrm{~b}$ compared with the IGM emission as a function of the distance from HCG $62 \mathrm{~b}$. The contamination from HCG $62 \mathrm{~b}$ becomes less than $10 \%$ of the IGM intensity when one goes to outside of a radius of $10^{\prime \prime}$. Thus, we extracted a circle with $10^{\prime \prime}$ radius around HCG $62 \mathrm{~b}$ and carried out the analysis for the IGM emission.

The central HCG 62 image is shown in figure 3 (a). This image indicates surface brightness depressions in the northeast and southwest directions at 20"-30" from HCG 62a, the so-called cavities as reported by Vrtilek et al. (2001,2002). We look into the properties of these cavities in $\S 4$. 
Table 3. Best fit parameters of the radial surface brightness profiles with ACIS-S3 $\left(r<4^{\prime}\right)$ and MOS1 $\left(1^{\prime}<r<14^{\prime}\right)$ in $0.5-4 \mathrm{keV}$ by the $3-\beta$ model. First and second components were constrained to have common $\beta$. The parameters of $\beta$ and $R_{\mathrm{c}}$ of the third component were fixed at the ROSAT result.

\begin{tabular}{|c|c|c|c|}
\hline & 1 & 2 & 3 \\
\hline$S_{0}\left(10^{-6}\right.$ photons $\left.\mathrm{cm}^{-2} \mathrm{~s}^{-1} \operatorname{arcsec}^{-2}\right)$ & $0.83_{-0.08}^{+0.10}$ & $0.29 \pm 0.03$ & $0.0018 \pm 0.0001$ \\
\hline$\ldots \ldots \ldots$ & $0.65 \pm 0.02$ & $\leftarrow$ (fixed) & 0.63 (fixed) \\
\hline$R_{\mathrm{c}}(\operatorname{arcmin} / \mathrm{kpc}) \ldots \ldots \ldots \ldots \ldots \ldots \ldots \ldots$ & $0.10_{-0.01}^{+0.02} / 1.7_{-0.2}^{+0.3}$ & $0.48_{-0.03}^{+0.04} / 8.5 \pm 0.6$ & 9.00/159.08 (fixed) \\
\hline$\chi^{2} /$ dof & & $755.39 / 570$ & \\
\hline
\end{tabular}

Table 4. Best fit parameters by the 2 -dimensional $2-\beta$ model for the central region $\left(r<1^{\prime}\right)$ of HCG 62 with ACIS-S3 in $0.5-4 \mathrm{keV}$.

\begin{tabular}{|c|c|c|}
\hline & 1 (narrower) & 2 (wider) \\
\hline$S_{0}\left(10^{-6}\right.$ photons $\left.\mathrm{cm}^{-2} \mathrm{~s}^{-1} \operatorname{arcsec}^{-2}\right)$ & $0.79_{-0.16}^{+0.21}$ & $0.63 \pm 0.06$ \\
\hline 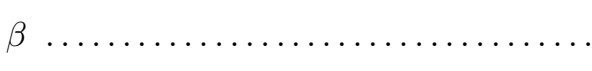 & $0.87_{-0.29}^{+0.86}$ & $0.44 \pm 0.01$ \\
\hline$R_{\mathrm{c}}(\operatorname{arcmin} / \mathrm{kpc}) \ldots$ & $0.07_{-0.03}^{+0.05} / 1.3_{-0.5}^{+0.9}$ & $0.18_{-0.01}^{+0.02} / 3.1 \pm 0.3$ \\
\hline R.A. $(J 2000) \ldots \ldots \ldots \ldots \ldots \ldots$ & $12^{\mathrm{h}} 53^{\mathrm{m}} 05.63 \pm 0.4^{\prime \prime}$ & $12^{\mathrm{h}} 53^{\mathrm{m}} 05.90 \pm 0.4^{\prime \prime}$ \\
\hline Dec. $(\mathrm{J} 2000) \ldots$.... & $-09^{\circ} 12^{\prime} 15^{\prime \prime} 5 \pm 0.4^{\prime \prime}$ & $-09^{\circ} 12^{\prime} 11 .^{\prime \prime} 6 \pm 0.3^{\prime \prime}$ \\
\hline$\chi^{2} /$ dof $\ldots .$. & \multicolumn{2}{|c|}{$132.07 / 122$} \\
\hline
\end{tabular}

\subsection{XMM-Newton image}

A combined X-ray image taken with MOS1 and MOS2 detectors of XMM-Newton in the 0.5-4 keV energy band is shown in figure 3 (b). We corrected for background and exposure, and performed Gaussian smoothing to the image. The IGM emission is very extended beyond the boundary of the Chandra ACIS-S3 chip shown by a white square. The cavities are not clearly seen because of the poorer spatial resolution. Many point sources were detected in the outer region by the SAS "edetect" task, but none of them outside of $r=100^{\prime \prime}$ were member galaxies of this compact group. Point sources inside of 100 " from HCG 62a were excluded using the Chandra data (§3.1), and the XMM-Newton data were used to mask out the sources outside of $100^{\prime \prime}$.

\subsection{Surface brightness profile}

We plot the radial surface brightness profile for the ACIS-S3 $\left(r<4^{\prime}\right)$ and MOS1 $\left(1^{\prime}<\right.$ $r<14^{\prime}$ ) around HCG $62 \mathrm{a}$ in the energy range of $0.5-4 \mathrm{keV}$ in figure 4 . In this analysis, only the MOS1 data were used for the XMM-Newton observation because of its low background and good spatial resolution. HCG $62 \mathrm{~b}$ was excluded with a circle of $10^{\prime \prime}$ radius. Background subtraction was carried out separately for each instrument. Mulchaey \& Zabludoff (1998) report an acceptable fit with a double $\beta$ model for the ROSAT data. As the first attempt, we fitted the radial profile with the same ROSAT model, however, a large discrepancy was found in the 


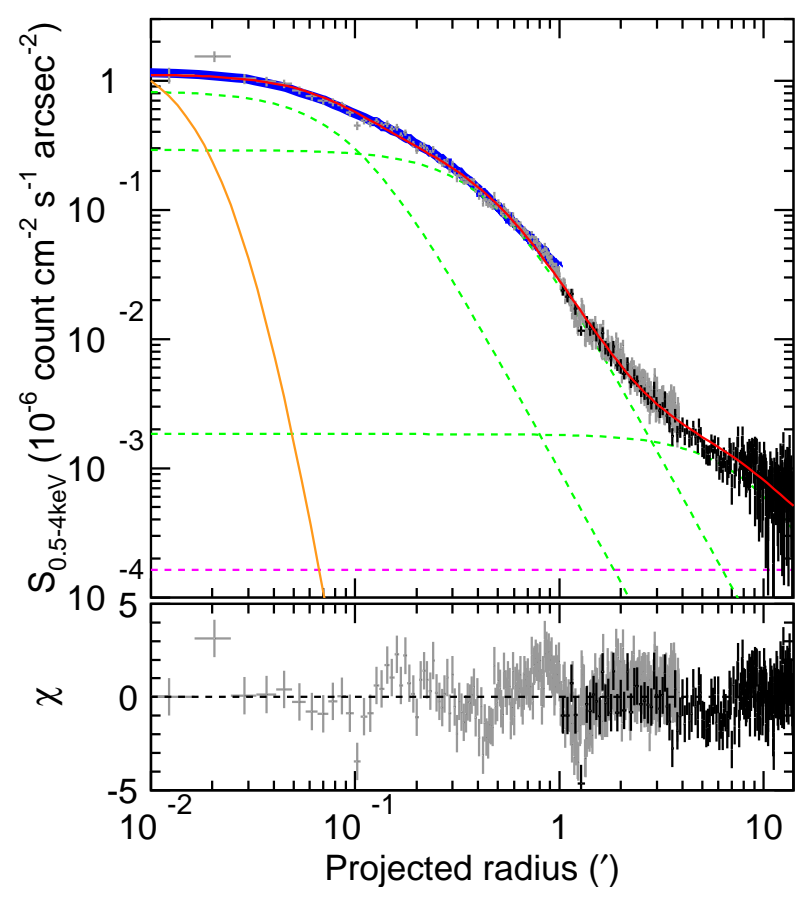

Fig. 4. In the upper panel, radial profiles of the surface brightness of HCG 62 in $0.5-4 \mathrm{keV}$ around the central galaxy HCG 62a are plotted for ACIS-S3 $\left(r<4^{\prime}\right)$ and MOS1 $\left(1^{\prime}<r<14^{\prime}\right)$, as indicated by grey and black lines, respectively. The orange solid line represents the point spread function of Chandra. The best-fit 3- $\beta$ model is shown with red solid line, and the three components are indicated with green dashed lines. For the outermost $\beta$ component, parameters of $\beta$ and $R_{\mathrm{c}}$ are fixed to the ROSAT result by Mulchaey \& Zabludoff (1998). Intensity for the sum of the soft Galactic and the hard emission is indicated by horizontal magenta dashed line. The blue thick line represents the projected best-fit 2-dimensional double $\beta$ model obtained with the Chandra image for the central region $\left(r<1^{\prime}\right)$. In the bottom panel, the residuals of the fit are shown in unit of $\sigma$.

central region around $r \lesssim 1^{\prime}$. We then varied the parameters of the inner $\beta$ component. The soft background and the hard component are included as fixed constants in the fit. The fit was still unacceptable because of a large discrepancy in the central region. Then, we fitted with a 3- $\beta$ model by adding a narrow component with its $\beta$ parameter constrained to have the same value with the middle component, and the fit was much improved. The outermost component was fixed to the ROSAT value, because the IGM emission of HCG 62 is much extended over the XMM-Newton field of view, and we could not constrain the parameters.

The fit results are summarized in table 3 . The derived core radius, $R_{\mathrm{c}}=0.48_{-0.03}^{+0.04}$, for the middle component are consistent with the value, $R_{\mathrm{c}}=0.56_{-0.16}^{+0.16}$, by Mulchaey \& Zabludoff (1998) with ROSAT PSPC, while their $\beta=0.79_{-0.09}^{+0.10}$ is steeper than ours, $\beta=0.65 \pm 0.02$. This is due certainly to the narrow $\beta$ model component we have introduced, which is required because of the superior angular resolution of Chandra ACIS-S3 than ROSAT PSPC. The obtained $\chi^{2}$ value is still large, however addition of yet another (4th) $\beta$ component does not significantly improve the fit, and the derived $\beta$ values for the inner two components are close to 3.0 which 
is obviously too steep. Therefore, we conclude that it is difficult to improve the fit better than the $3-\beta$ model case described above, due mainly to the complicated spatial structure in the central region.

We next carried out a 2-dimensional fitting for the Chandra X-ray image. The ACIS-S3 image shown in figure 3 (a) suggests that the brightest position may be slightly offset from the center of the extended group gas. To examine this, we fitted the 2-dimensional image in 0.5-4 keV within $r<1^{\prime}$ around HCG 62a with a 2- $\beta$ model (narrower and wider), whose centers were varied as free parameters. Due to the limited photon count in the image bin, we chose the maximum likelihood method assuming the Poisson statistics in the fitting. The "blank-sky" background were included in the model as a constant surface brightness. The data and the best fit model are projected around the common center (HCG 62a) as shown in figure 4. The model (blue thick line) well describes the radial profile within $r \leq 1^{\prime}$, and gives an acceptable fit with $\chi^{2} /$ dof $=132.07 / 122$. The fit parameters are summarized in table 4 . We find that the centers of the narrower and wider components are different by $5.44^{\prime \prime}(1.6 \mathrm{kpc})$. The center of the narrower component is closer to the optical center ( $2^{\prime \prime}$ offset) than the wider component ( $5^{\prime \prime}$ offset).

\section{X-ray Cavity}

In this section, we look into the X-ray structure and the spectral characteristics of the cavities which are recognized in the Chandra image in figure 3 (a). We broadly consider two possibilities for the origin of the cavities as follows; (1) depression of the X-ray flux is resulted by absorption due to some intervening material, and (2) X-ray emitted gas is deficient, possibly being expelled by some process. We will examine the case (1) in $\S 4.2$ and (2) in $\S 4.3$, respectively.

\subsection{Flux depression 65 temperature map}

To quantify surface brightness depression in the cavity regions, we refer to the result of the 2 - $\beta$ model fit of the 2 -dimensional image in $\S 3.3$. The relative deviation of the brightness from the $2-\beta$ model is color coded and plotted in figure 5 . Both of the cavity regions show a brightness drop by as much as 50-70\% at the bottom as compared with the level of surrounding regions. If we approximate the cavity shape by a sphere, their radii are $\sim 10^{\prime \prime}(3 \mathrm{kpc})$ as indicated by white circles in figure 5 , with the north one slightly larger. This approximation is similar to the one performed in B04. The distances of the cavity centers from HCG 62a are $\sim 30^{\prime \prime}$ (9 kpc) for the north and $\sim 20^{\prime \prime}(6 \mathrm{kpc})$ for the south one, respectively. If we take the distances from the central position of the wider component of the $2-\beta$ model, the cavities are almost symmetrical in their distances $\sim 25^{\prime \prime}(7.4 \mathrm{kpc})$. The positions and the distances of both cavities are summarized in table 5 .

Next, we look into temperature distribution using hardness ratios $(H R)$. After the point 

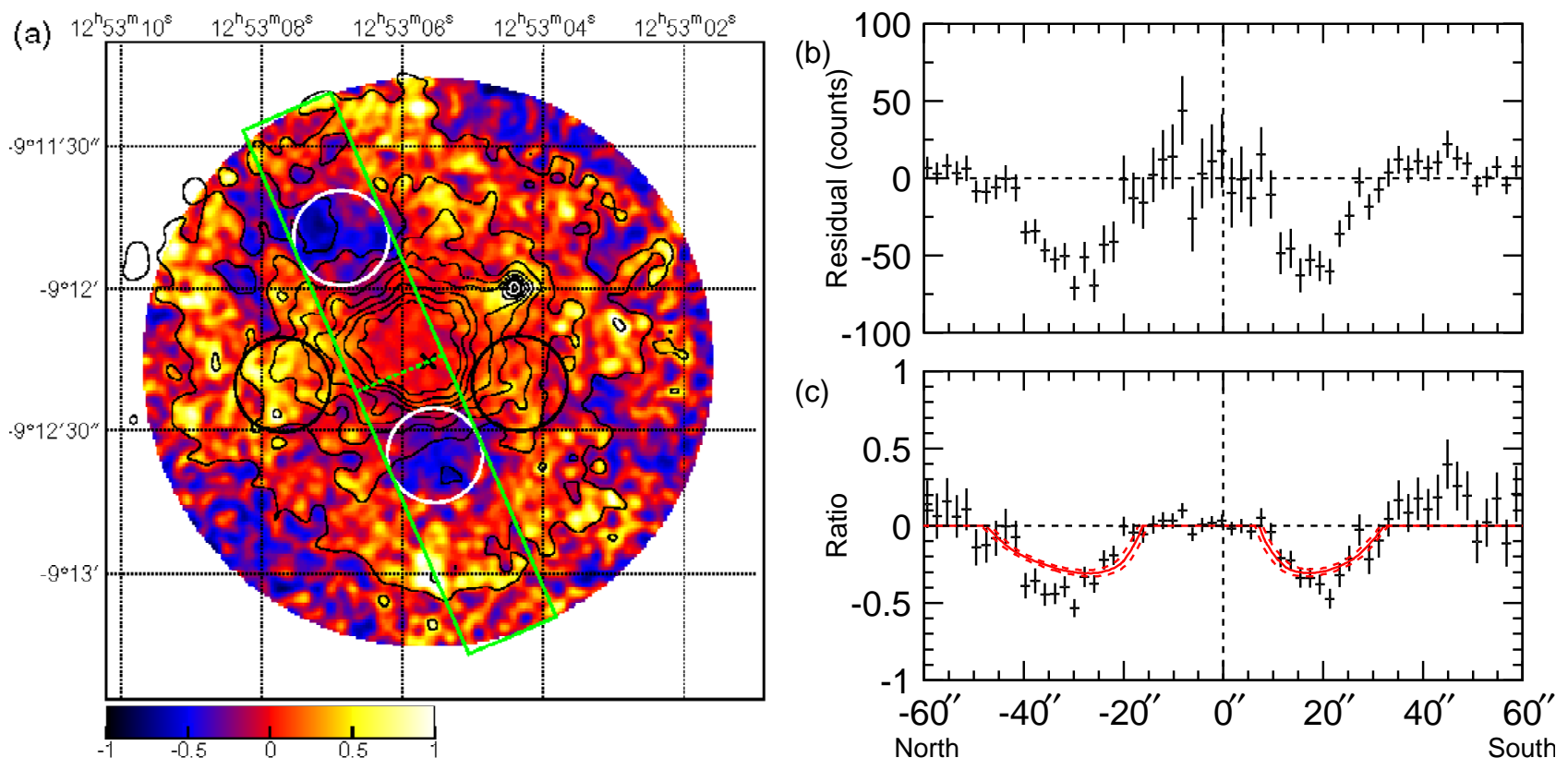

Fig. 5. (a) Color-coded map of the relative deviation of the flux from the best-fit 2- $\beta$ model based on the 2-dimensional fit (table 4) in $0.5-4 \mathrm{keV}$ within a radius of $1^{\prime}$ around HCG 62a with ACIS-S3 (J2000). $\mathrm{X}$-ray contours shown by think black lines are the same in figure 3 (a). White and black circles with a radius of $10^{\prime \prime}$ represent regions where energy spectra are studied. White ones are the cavities, and black ones are non-cavity regions examined for comparison. The distances from HCG $62 \mathrm{a}$ to the centers of the east and west light-blue circles are $20^{\prime \prime}$ and $30^{\prime \prime}$, the same for the north and south cavities, respectively.

(b) Residual of the observed count to the $2-\beta$ model along the green rectangular region $\left(20^{\prime \prime} \times 120^{\prime \prime}\right)$ in (a). (c) Ratio of the residual counts divided by the $2-\beta$ model. See text $\S 4.1$ for red lines.

source and background subtractions, we produced images in two energy bands, $0.5-0.95 \mathrm{keV}$ $(S)$ and $0.95-4 \mathrm{keV}(H)$, which give nearly the same counts in both energy bands. The $H R$ is defined as the ratio of the counts between these bands as $H R \equiv(H-S) /(H+S)$. We divided the region into small cells whose sizes are determined to contain at least 50 counts in the $0.5-4.0 \mathrm{keV}(H+S)$ band, and then the $H R$ values were calculated. The $H R$ values were then converted to temperature based on the response matrices at the center of the field of view, assuming an absorption of $N_{\mathrm{H}}=3.0 \times 10^{20} \mathrm{~cm}^{-2}$ and a metal abundance of 0.5 solar. Typical $90 \%$ confidence error on $H R$ is $\sim 0.17$, which corresponds to $\sim 0.2 \mathrm{keV}$ above $1 \mathrm{keV}$ and $\sim 0.1 \mathrm{keV}$ below $1 \mathrm{keV}$. The resultant temperature map is shown in figure 6 . A clear temperature drop down to $\sim 0.7 \mathrm{keV}$ is seen at the central region, which is surrounded by a hotter gas with $k T \sim 1.4 \mathrm{keV}$ at a radius greater than $1^{\prime}(18 \mathrm{kpc})$. The central cool region shows an irregular shape, with an elongation to the directions of two cavities, although the temperature structure does not simply correlate with the cavity regions. 


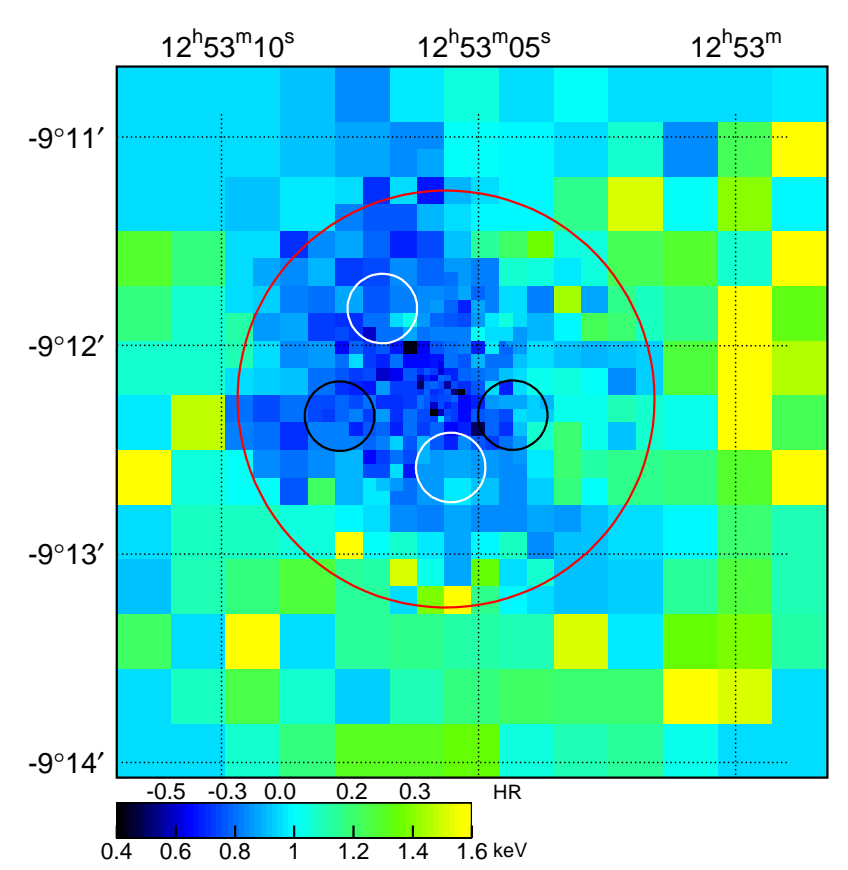

Fig. 6. Color coded temperature map (J2000) based on the hardness ratio, $H R \equiv(H-S) /(H+S)$, with ACIS-S3, where $S$ and $H$ correspond to the $0.5-0.95 \mathrm{keV}$ and $0.95-4 \mathrm{keV}$ counts, respectively. The red circle has a radius of $1^{\prime}$ around HCG $62 \mathrm{a}$, and the small circles $\left(10^{\prime \prime}\right.$ in radius) are the same as those in figure 5. Typical $90 \%$ confidence error on temperature is $\sim 0.2 \mathrm{keV}$ above $1 \mathrm{keV}$ and $\sim 0.1 \mathrm{keV}$ below $1 \mathrm{keV}$.

\subsection{Spectral comparison between cavity $\&$ non-cavity regions}

In order to examine the possibility that absorption may be responsible for the cavities, we look into the energy spectra in this subsection. We extracted energy spectra for the two cavity regions from the ACIS-S3 data, using circular regions with the same radius of $10^{\prime \prime}$ (3 kpc) as shown in figure 5. To compare with them, we also extracted spectra for two non-cavity regions with both $10^{\prime \prime}$ radii in the east and west at the same distance from HCG 62 a to the respective cavities. Their precise locations are $\left(12^{\mathrm{h}} 53^{\mathrm{m}} 06.87,-9^{\circ} 11^{\prime} 49^{\prime \prime} 4\right),\left(12^{\mathrm{h}} 53^{\mathrm{m}} 05.54,-9^{\circ} 12^{\prime} 35^{\prime \prime} 1\right)$, $\left(12^{\mathrm{h}} 53^{\mathrm{m}} 07^{\mathrm{s}} .75,-9^{\circ} 12^{\prime} 21^{\prime \prime} 1\right)$ and $\left(12^{\mathrm{h}} 53^{\mathrm{m}} 04^{\mathrm{s}} 33,-9^{\circ} 12^{\prime} 20^{\prime \prime} 1\right)$ (J2000) for the north, south cavities and east, west non-cavity, respectively. The non-cavity regions are indicated by black circles in figure 5, and their properties are summarized in table 5. First of all, we calculated the hardness, $H R$, as defined in the previous subsection, in these four regions. The derived values with $90 \%$ confidence errors were $-0.130 \pm 0.066$ vs. $-0.209 \pm 0.052$ for the north cavity vs. east noncavity pair, and $-0.099 \pm 0.058$ vs. $-0.117 \pm 0.046$ for the south cavity vs. west non-cavity pair, respectively. There is no significant difference in the hardness for both pairs. However, both of the cavity regions suggest slightly larger hardness than the respective non-cavity regions, which is in the same sense as expected if absorption is responsible for the X-ray cavities.

To be more quantitative, we conducted a spectral fit for each spectrum. All the spectra were binned to contain at least 30 counts in a bin. The spectra were then fitted with a two 
Table 5. Observed and calculated properties of the north and south cavities and the east and west non-cavities.

\begin{tabular}{|c|c|c|c|c|}
\hline & north cavity & east non-cavity & south cavity & west non-cavity \\
\hline Distance from HCG $62 \mathrm{a}^{*} \ldots \ldots \ldots$ & $31.9^{\prime \prime}$ & $31.9^{\prime \prime}$ & $19.7^{\prime \prime}$ & $19.8^{\prime \prime}$ \\
\hline Distance from group core ${ }^{\dagger}$ & $26.5^{\prime \prime}$ & $29.0^{\prime \prime}$ & $24.1^{\prime \prime}$ & $24.7^{\prime \prime}$ \\
\hline$H R^{\ddagger}$ & $-0.13 \pm 0.07$ & $-0.21 \pm 0.05$ & $-0.10 \pm 0.06$ & $-0.12 \pm 0.05$ \\
\hline Integration radius ${ }^{\S} \ldots \ldots \ldots \ldots \ldots$ & $15.7^{\prime \prime}$ & $\leftarrow$ & $12.6^{\prime \prime}$ & $\leftarrow$ \\
\hline$N_{\mathrm{c}}$ or $N_{\mathrm{nc}} \| \ldots \ldots \ldots \ldots$ & $2225 \pm 48$ & $2857 \pm 58$ & $2476 \pm 63$ & $3316 \pm 75$ \\
\hline$F_{\text {proj }}-F_{\text {sphere }}$ or $F_{\text {proj }} \sharp$ & 50.63 & 64.93 & 48.31 & 63.46 \\
\hline
\end{tabular}

* Position of HCG 62a is assumed to be coincide with the center of the narrower component in table 4.

$\dagger$ Position of the group core is assumed to be coincide with the center of the wider component in table 4.

$\ddagger$ Hardness ratio, $H R \equiv(H-S) /(H+S)$, within $r \leq 10^{\prime \prime}$, where $S$ (or $\left.H\right)$ corresponds to 0.5-0.95 (0.95-4) keV counts.

$\S$ Integration radius is chosen to become $N_{\mathrm{c}} / N_{\mathrm{nc}}=\left(F_{\text {proj }}-F_{\text {sphere }}\right) / F_{\text {proj }}$ for each cavity and non-cavity pair.

$\| N_{\mathrm{c}}$ for north and south cavities, and $N_{\mathrm{nc}}$ for non-cavities. Each value is corrected for background and exposure.

$\sharp F_{\text {proj }}-F_{\text {sphere }}$ for north and south cavities, and $F_{\text {proj }}$ for non-cavities in arbitrary unit.

Table 6. Results of spectral fits for the north and south cavity regions and the east and west non-cavity regions within $r \leq 10^{\prime \prime}$ in $0.5-3 \mathrm{keV}$ with a two temperature vMekal model. See text $\S 4.2$ for details.

\begin{tabular}{|c|c|c|c|c|c|c|}
\hline & $\begin{array}{c}N_{\mathrm{H}, \text { excess }} \\
\left(10^{20} \mathrm{~cm}^{-2}\right)\end{array}$ & $\begin{array}{l}k T_{1} \\
(\mathrm{keV})\end{array}$ & $\begin{array}{r}\text { Norm }_{1}{ }^{*} \\
\left(10^{-19}\right.\end{array}$ & $\begin{array}{l}\mathrm{Norm}_{2}{ }^{*} \\
\left.\mathrm{~cm}^{-5}\right)\end{array}$ & $\begin{array}{c}F_{0.5-4 \mathrm{keV}} \\
\left(10^{-14} \mathrm{ergs}\right.\end{array}$ & $\begin{array}{r}\chi^{2} / \text { dof } \\
\left.\mathrm{cm}^{-2} \mathrm{~s}^{-1}\right)\end{array}$ \\
\hline \multicolumn{7}{|c|}{ phabs $\left(N_{\mathrm{H}}=3.0 \times 10^{20} \mathrm{~cm}^{-2}\right.$ fixed $) \times 2-T\left(k T_{2}=1.4 \mathrm{keV} \&\right.$ abundance fixed $)$ vMekal model } \\
\hline north cavity $\ldots \ldots \ldots \ldots \ldots \ldots \ldots$ & - & $0.74_{-0.05}^{+0.04}$ & $1.3_{-0.2}^{+0.3}$ & $0.7_{-0.4}^{+0.4}$ & $4.0 \pm 0.3$ & $18 / 14$ \\
\hline east non-cavity & - & $0.75_{-0.05}^{+0.04}$ & $2.5_{-0.4}^{+0.4}$ & $0.6_{-0.5}^{+0.5}$ & $6.8 \pm 0.4$ & $22 / 16$ \\
\hline south cavity ... & - & $0.76_{-0.06}^{+0.05}$ & $1.6_{-0.3}^{+0.3}$ & $1.1_{-0.4}^{+0.6}$ & $5.2 \pm 0.3$ & $18 / 20$ \\
\hline west non-cavity $\ldots \ldots \ldots \ldots \ldots \ldots$ & - & $0.76_{-0.05}^{+0.04}$ & $2.6_{-0.4}^{+0.4}$ & $1.3_{-0.7}^{+0.7}$ & $8.0 \pm 0.4$ & $39 / 32$ \\
\hline \multicolumn{7}{|c|}{ phabs $\left(N_{\mathrm{H}}=3.0 \times 10^{20} \mathrm{~cm}^{-2}\right.$ fixed $) \times$ zphabs $\times 2-T\left(k T_{2}=1.4 \mathrm{keV} \&\right.$ abundance fixed $)$ vMekal model } \\
\hline north cavity $\ldots \ldots \ldots \ldots \ldots \ldots \ldots$ & $18.3_{-4.6}^{+5.6}$ & $0.70_{-0.02}^{+0.06}$ & $2.5_{-0.4}^{+0.6}$ & $0.7_{-0.3}^{+0.3}$ & $4.2 \pm 0.3$ & \multirow{2}{*}{$42 / 31$} \\
\hline east non-cavity ... & $2.4_{-2.4}^{+3.3}$ & $\uparrow$ & $\uparrow$ & $\uparrow$ & $6.5_{-0.3}^{+0.4}$ & \\
\hline south cavity $\ldots \ldots \ldots \ldots \ldots \ldots \ldots$ & $11.7_{-2.3}^{+3.6}$ & $0.75_{-0.04}^{+0.03}$ & $2.5_{-0.3}^{+0.4}$ & $1.2_{-0.4}^{+0.4}$ & $5.5 \pm 0.3$ & \multirow{2}{*}{$76 / 53$} \\
\hline west non-cavity ... & $<2.8$ & $\uparrow$ & $\uparrow$ & $\uparrow$ & $7.6 \pm 0.4$ & \\
\hline
\end{tabular}

* Normalization for the Mekal model, Norm $=\int n_{\mathrm{e}} n_{\mathrm{H}} d V /\left(4 \pi(1+z)^{2} D_{\mathrm{A}}^{2}\right)$, where $D_{\mathrm{A}}$ is the angular distance to the source.

temperature vMekal model (2-T model in $\S 5)$ in the $0.5-3 \mathrm{keV}$ band with an absorption (phabs in XSPEC) fixed to the Galactic value, $N_{\mathrm{H}}=3.0 \times 10^{20} \mathrm{~cm}^{-2}$. Because of the limited statistics of those spectra, temperature of the hot component, $k T_{2}$, was fixed to $1.4 \mathrm{keV}$ and the abundance of each element was fixed to the best-fit values for the 1- $T$ model in the annulus range of $0.4<r<0.6^{\prime}$ in table 7 . See $\S 5$ for details of the model. The fit results are summarized in the first four rows of table 6 . 


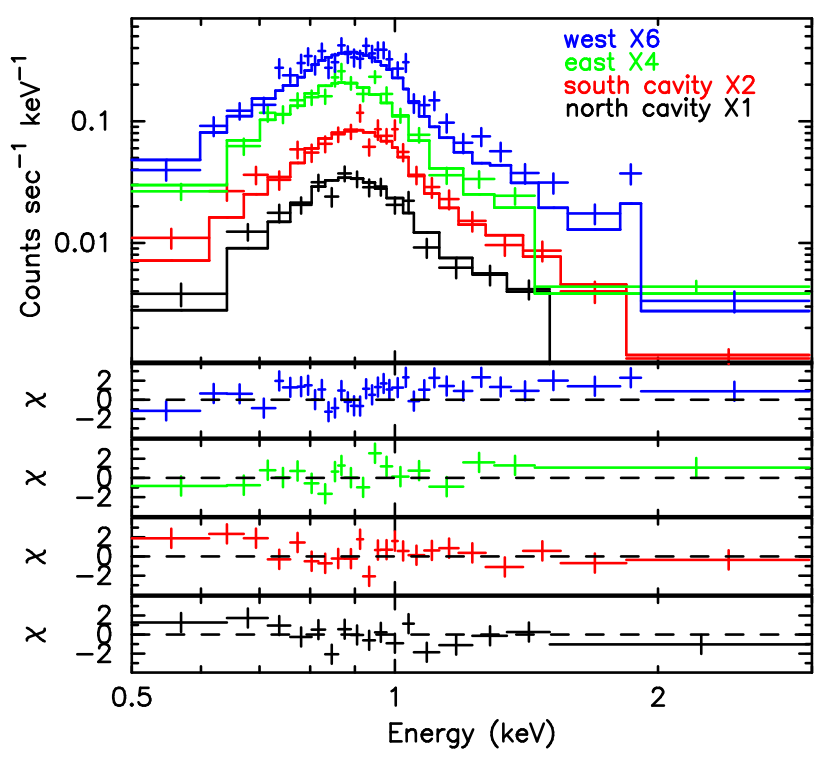

Fig. 7. Chandra ACIS-S3 X-ray spectra for north (black) and south (red) cavity regions, and east (green) and west (blue) non-cavity regions, respectively. Spectra of the north cavity and east non-cavity pair and the south cavity and east non-cavity pair are simultaneously fitted with common continuum parameters with individual excess absorption. The spectra and the best-fit models are scaled by 2, 4, 6 for south, east, and west regions, respectively, for clarity. The bottom four panels show the residuals of the fit.

All of the four spectra are well fitted by the $2-T$ model with acceptable $\chi^{2}$ values at the $90 \%$ confidence limit. The obtained temperatures exhibited quite similar values of $k T_{1}=0.74-$ $0.76 \mathrm{keV}$, and we could not find any significant difference between the cavity and non-cavity spectra except for the normalizations of the cool component, $\mathrm{Norm}_{1}$. It is interesting that the normalizations of the hot component, $\mathrm{Norm}_{2}$, are not different between them. As described in the next section, the hot component is probably absent from the central region of the group $\left(r \lesssim 0.6^{\prime}\right)$, so that the hot component seen in these four regions are mostly due to foreground or background emission. It is therefore suggested that the cavities lie close to the group core in our line of sight, and that only the cool component is pushed away from the cavities. The signature of hardening of $H R$ above is due probably to this effect. As a remark, the fits are not acceptable when fitted with a one temperature vMekal model (1- $T$ model), although the obtained temperatures are also very similar among the four spectra.

Here, we evaluate how much $N_{\mathrm{H} \text {,excess }}$ is needed to reproduce the observed cavity feature by a simple absorption at the source redshift. For this purpose, we simultaneously fitted the cavity and non-cavity spectra of each pair, by setting all the parameters of the emission spectrum to be the same including the normalization. Thus, two new parameters, $N_{\mathrm{H} \text {,excess }}$ for each pair, and three new constraint on $\mathrm{Norm}_{1}, \mathrm{Norm}_{2}$, and $k T_{1}$ are added, so that the degree of freedom (dof) increases by one. The pulse-height spectra and fit residuals for the four regions are shown in figure 7 . The $N_{\mathrm{H} \text {,excess }}$ for the individual regions are summarized in the bottom four rows of table 6 . The obtained $\chi^{2} /$ dof were $42 / 31$ for the north cavity and east non-cavity 
pair, and 76/53 for the south cavity and west non-cavity pair. Therefore both fits were not acceptable at the $90 \%$ confidence limit, although improvement of $\chi^{2}$ is marginal for the former pair. The derived $N_{\mathrm{H} \text {,excess }}$ in cavities has to be $\sim 3$ times larger than those in non-cavity regions for the necessary flux reduction. The required mass of neutral hydrogen for north and south cavities amount to $1.0 \times 10^{9} M_{\odot}$ and $0.4 \times 10^{9} M_{\odot}$, respectively. These values are comparable to the observed upper limit of the $\mathrm{H}_{\mathrm{I}}$ mass, $\sim 10^{9} M_{\odot}$, of the whole group including all member galaxies by Verdes-Montenegro et al. (2001) and Stevens et al. (2004). It is therefore difficult to attribute the cavities to the absorption by neutral gas.

\subsection{Hollow sphere model}

Since the absorption model is found to be unlikely in the previous subsection, we then consider an extreme case that a spherical cavity contains no X-ray emitting gas at all, namely, the hollow sphere model. It is assumed that the spherical cavities exist at the same distance from us to the group core. Even with this extreme assumption, there should be a certain flux observed in the projected circular cavity region because of the foreground and background group emission. This will give us a constraint on the physical size of the cavities. For an overall $\mathrm{X}$-ray structure, we assume that the 3 -dimensional X-ray emissivity obeys the 3 - $\beta$ model shown in table 3. Based on this model, we can estimate the emission from an arbitrary volume in the IGM and the emission from a column integrated along a certain line of sight. Here a single cavity is considered. The observed projected counts corrected for background and exposure at the circular cavity region is denoted by $N_{\mathrm{c}}$, and the count in the corresponding non-cavity region by $N_{\mathrm{nc}}$, respectively. The non-cavity region has the same integration radius and distance from HCG $62 \mathrm{a}$ as the cavity region. Then, based on the $3-\beta$ model, we can calculate the flux, $F_{\text {sphere }}$, from the cavity volume which is assumed to be filled with hot gas. The model also gives us the projected flux, $F_{\text {proj }}$, from the line-of-sight column passing through the non-cavity region. These numbers allow us to estimate the counts purely emitted from the spherical cavity as, $N_{\mathrm{c}}-N_{\text {nc }}\left(F_{\text {proj }}-F_{\text {sphere }}\right) / F_{\text {proj }}$, which should be zero on the assumption above.

Based on the observed cavity flux, we can derive the upper and lower limits for the radius of the hollow spheres. Clearly, too small radius will give too little depression in the $\mathrm{X}$-ray flux at the cavity region, and vise versa. We applied the observed intensity and position of the north and south cavities. The allowed ranges for the radii of the hollow spheres were computed as $r=15.7 \pm 0.9^{\prime \prime}$ and $r=12.6 \pm 0.8^{\prime \prime}$ for the north and south cavities, respectively. These sizes are almost consistent with the projected image as seen in figure 5 (a). The observed and the calculated values of $N_{\mathrm{c}}, N_{\mathrm{nc}}, F_{\text {proj }}-F_{\text {sphere }}$, and $F_{\text {proj }}$ are summarized in table 5 . The expected profile of the count rate deficiency with this assumption is overlaid in figure 5 (c) as a solid red line. The dashed red lines correspond to the radii at the $\pm 90 \%$ confidence errors. As seen in this plot, the spherical assumption of cavities gives an asymmetric shape with deeper deficiency at the near side and shallower at the far side, when projected to the 2-dimensional 
image. Because the observed shapes of the cavities are almost symmetrical, it is suggested that there must be an asymmetry in the shape and/or the density inside the cavities. Namely, the far-side of the cavities should be larger in size and/or weaker in the X-ray emissivity. It is also notable that even with this extreme assumption of hollow spheres at the same distance to the group core, the calculated deepness of the deficiency is nearly the same level or even slightly shallower than the observed ones. This fact implies that the shape of cavities is probably elongated in the direction of our line of sight. Another possibility is that the group gas itself has a flatter shape in the depth, although it is implausible considering the redshift distribution of the member galaxies (Zabludoff \& Mulchaey 2000) and that the projected X-ray image is quite symmetric.

\section{Radial Profiles of Temperature \& Abundance}

\subsection{Consistency between Chandra \& XMM-Newton}

In order to obtain radial profiles of temperature and abundance of the IGM, we examined energy spectra for each instrument (ACIS-S3, MOS1, MOS2, and pn) from several circular annuls from the center of HCG 62a. The center was taken from the narrower component in our 2 -dimensional fit carried out in $\S 3.3$. First of all, we checked the consistency between Chandra and XMM-Newton using the spectra within $r \leq 2^{\prime}$ around HCG 62a. Each spectrum was binned to contain at least 30 counts per bin to be tested with the $\chi^{2}$ fit. The background data were taken from the same region in the "blank-sky" data for each instrument. The hard emission and the soft background components are also considered in the fitted model ( $\S 2.3$ ). As for the XMM-Newton data, MOS1 and MOS2 spectra were summed up and simultaneously fitted

with the pn spectrum. The energy range around the Al- $\mathrm{K}_{\alpha}$ line $(1.4867 \mathrm{keV})$, the instrumental background, was ignored for both MOS and pn.

We have fitted the spectra with a single temperature or two temperature vMekal model including the Galactic photoelectric absorption. Abundances of $\mathrm{C}, \mathrm{N}, \mathrm{Na}$, and $\mathrm{Al}$ were fixed to be 1 solar. We grouped several elements and constrained them to have a common abundance. The first group contains $\mathrm{O}$ and Ne, the second group S, Ar and Ca, and the third group is for Fe and Ni. Among the other elements, abundances of Mg and Si were determined separately. In the case of the two temperature fit, we used the sum of two vMekal models in which two components were constrained to have common abundances. The actual model formula are phabs $\times($ vMekal + powerlaw $)+$ Mekal $(1-T$ model $)$ and phabs $\times($ vMekal + vMekal + powerlaw $)$ + Mekal (2-T model). Here, phabs represents photoelectric absorption and $N_{\mathrm{H}}$ is fixed at the Galactic value of $3.0 \times 10^{20} \mathrm{~cm}^{-2}$. The Mekal component is the soft background described in $\S 2.3$, with $k T$ and $Z$ both fixed at $0.3 \mathrm{keV}$ and 1 solar, respectively, and with the fixed normalization. The power-law represents the hard emission described in $\S 2.3$ with its photon index, $\Gamma=1.5$, and normalization both fixed. 
The $r \leq 2^{\prime}$ spectra are shown in figure $8(\mathrm{a})$, and the fit results with $2-T$ model are summarized in table 7 . The $1-T$ model was rejected at high significance with $\chi^{2} /$ dof $=2162 / 425$ (combined) for this region. Three kinds of the fit results are listed in table 7, Chandra (ACISS3) only, XMM-Newton (MOS1+2, pn) only, and the combined fit of all the instruments. We have confirmed that most of the results from different instruments were consistent at the $95 \%$ confidence limit. The abundances with ACIS-S3 were slightly larger than, but mostly consistent with those with XMM-Newton, while only Si gave inconsistent values at the $95 \%$ confidence limit. This is due probably to the systematic effect by the intrinsic Si line as seen in the "blank-sky" data for both Chandra and XMM-Newton. Since the Si line is most prominent in the IGM spectrum, the good statistics result in formally inconsistent abundance values. In the annular range of $2-4^{\prime}$, spectral fit for individual instruments (ACIS-S3, MOS1+2, and pn) gave consistent results at the $90 \%$ confidence limit, due mainly to the poorer statistics. Based on these results, data from all the instruments were simultaneously fitted in the following analysis.

\subsection{Mekal vs APEC model \& notes on abundance}

We also summarize results when the spectra are fitted with phabs $\times(\mathrm{vAPEC}+\mathrm{vAPEC}+$ powerlaw $)+$ Mekal (2-T vAPEC model) in table 7. The vAPEC model (v1.3.1) gives smaller $\chi^{2}$ value especially for the Chandra spectrum, and indicates slightly higher temperatures by $\Delta T \lesssim 0.05 \mathrm{keV}$ than the vMekal model. This is due probably to the better modeling of the Fe-L line complex in the vAPEC model, however it is confirmed that all the element abundances are consistent within $90 \%$ confidence errors between the two models. We therefore adopt the vMekal model in the following analysis considering comparison with previous literatures.

Table 8 represents a result when all the element abundances in the vMekal or vAPEC model are determined separately for the combined fit. The errors of the $\mathrm{Ne}, \mathrm{Al}, \mathrm{Ar}, \mathrm{Ca}$, and $\mathrm{Ni}$ abundances are significantly larger than those of other elements, while other parameters agree with the values in table 7 within $90 \%$ confidence errors. Thus abundances in table 7 , which are linked together for $(\mathrm{O}, \mathrm{Ne}),(\mathrm{Mg}, \mathrm{Al}),(\mathrm{S}, \mathrm{Ar}, \mathrm{Ca})$, and $(\mathrm{Fe}, \mathrm{Ni})$, are essentially representing $\mathrm{O}$, $\mathrm{Mg}, \mathrm{S}$, and Fe abundances, respectively. There is an indication that the Ni abundance is higher than the Fe abundance in both vMekal and vAPEC model fits. This might be explained by the fact that the fitted spectra are not deprojected so that they contain emission from outer region in the group which exhibits higher temperature than the group core as seen in $\S 5.4$. On the other hand, it is known that $\mathrm{Ni}$ is synthesized more abundantly than Fe in SN Ia when compared in the solar unit, which will be discussed in $\S 7$.

\subsection{Deprojection analysis}

We conducted a deprojection analysis using energy spectra from several circular annuls from the center of HCG $62 \mathrm{a}$. The inner and outer radii of the annuls were $r=0-0.2^{\prime}, 0.2^{-}$ $0.4^{\prime}, 0.4-0.6^{\prime}, 0.6-1^{\prime}, 1-2^{\prime}, 2-4^{\prime}, 4-8^{\prime}$, and 8-14', in which $r$ represents the projected radius. The

Chandra ACIS-S3 data were used in the range of $r \leq 4^{\prime}$, and the XMM-Newton data were used in 

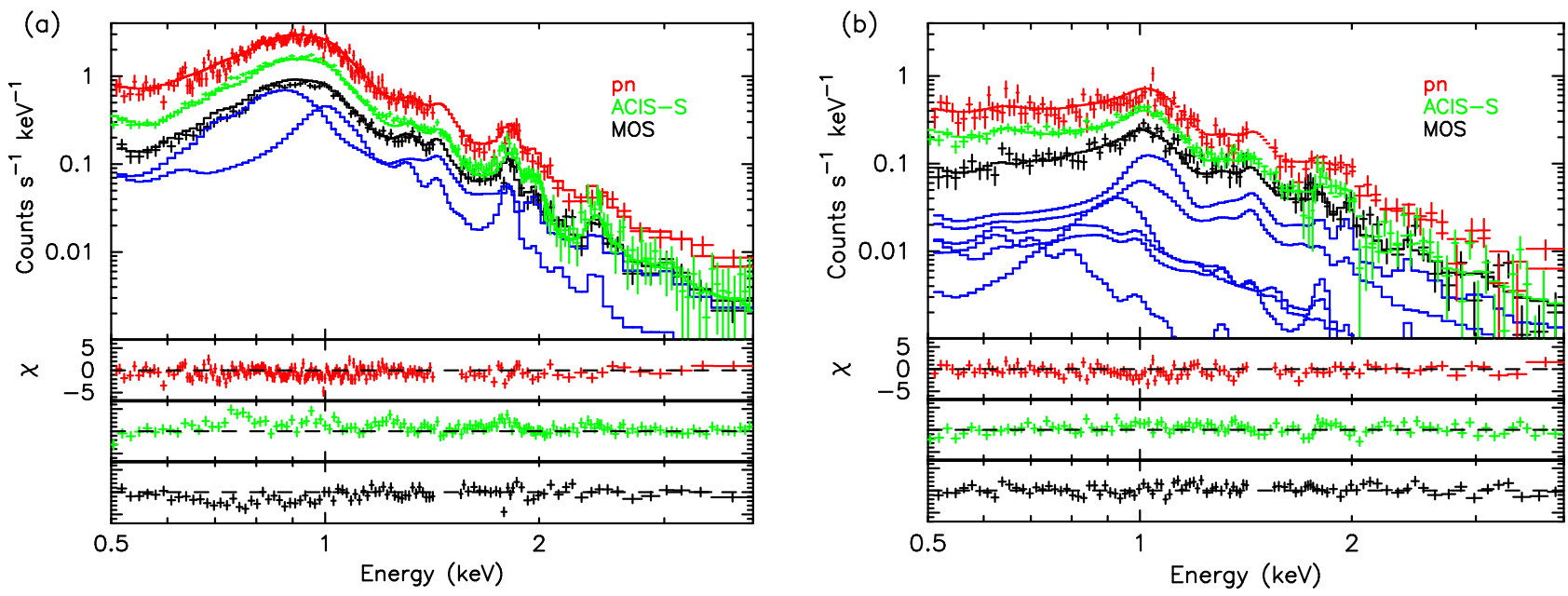

Fig. 8. (a) Chandra ACIS-S3 (green), MOS1+2 (black), and pn (red) spectra within $r \leq 2^{\prime}$ around HCG $62 \mathrm{a}$ in the $0.5-4 \mathrm{keV}$ band. The three spectra are simultaneously fitted with $2-T$ model without deprojection, and the best-fit models are indicated by solid lines. Each component of the two temperature vMekal model for MOS1+2 is indicated by blue lines. The bottom three panels show the residuals of the fit. (b) Same as (a), but in the 2-4' annulus fitted with the deprojected 2- $T$ model. The outer contributions and fitting models for MOS1+2 are indicated by blue lines.

Table 7. Results of the projected spectral fit for $r \leq 2^{\prime}$ region with two temperature vMekal and vAPEC models.

\begin{tabular}{|c|c|c|c|c|c|c|c|c|c|c|}
\hline & $\begin{array}{l}k T_{1} \\
(\mathrm{keV})\end{array}$ & $\begin{array}{l}k T_{2} \\
(\mathrm{keV})\end{array}$ & $\begin{array}{l}\mathrm{O}, \mathrm{Ne} \\
\text { (solar) }\end{array}$ & $\begin{array}{l}\mathrm{Mg}, \mathrm{Al} \\
\text { (solar) }\end{array}$ & $\begin{array}{l}\mathrm{Si} \\
\text { (solar) }\end{array}$ & $\begin{array}{l}\mathrm{S}, \mathrm{Ar}, \mathrm{Ca} \\
\text { (solar) }\end{array}$ & $\begin{array}{l}\mathrm{Fe}, \mathrm{Ni} \\
\text { (solar) }\end{array}$ & $\begin{array}{r}\text { Norm }_{1}{ }^{*} \\
\left(10^{-18}\right.\end{array}$ & $\begin{array}{l}\mathrm{Norm}_{2}{ }^{*} \\
\left.\mathrm{~cm}^{-5}\right)\end{array}$ & $\chi^{2} /$ dof \\
\hline \multicolumn{11}{|l|}{ vMekal } \\
\hline Chandra & $0.72_{-0.02}^{+0.02}$ & $1.32_{-0.06}^{+0.05}$ & $0.36_{-0.13}^{+0.12}$ & $1.20_{-0.19}^{+0.23}$ & $1.23_{-0.14}^{+0.19}$ & $0.86_{-0.29}^{+0.38}$ & $0.89_{-0.08}^{+0.09}$ & $6.6_{-1.0}^{+1.2}$ & $9.3_{-1.3}^{+1.3}$ & $189 / 144$ \\
\hline XMM & $0.77_{-0.05}^{+0.02}$ & $1.41_{-0.11}^{+0.12}$ & $0.23_{-0.13}^{+0.17}$ & $1.01_{-0.30}^{+0.37}$ & $0.76_{-0.15}^{+0.19}$ & $0.67_{-0.23}^{+0.26}$ & $0.81_{-0.12}^{+0.16}$ & $7.2_{-1.7}^{+1.8}$ & $8.5_{-1.3}^{+2.0}$ & $316 / 270$ \\
\hline Chandra & $0.77_{-0.02}^{+0.02}$ & $1.33_{-0.07}^{+0.15}$ & $0.41_{-0.13}^{+0.19}$ & $1.20_{-0.19}^{+0.22}$ & $1.18_{-0.15}^{+0.17}$ & $1.21_{-0.34}^{+0.37}$ & $0.91_{-0.08}^{+0.14}$ & $7.7_{-0.9}^{+0.9}$ & $7.8_{-1.1}^{+0.3}$ & $158 / 144$ \\
\hline XMM & $0.79_{-0.01}^{+0.01}$ & $1.47_{-0.11}^{+0.11}$ & $0.30_{-0.22}^{+0.34}$ & $0.97_{-0.32}^{+0.44}$ & $0.76_{-0.17}^{+0.23}$ & $0.71_{-0.25}^{+0.29}$ & $0.85_{-0.16}^{+0.22}$ & $7.4_{-1.9}^{+2.1}$ & $7.7_{-1.0}^{+1.0}$ & $311 / 270$ \\
\hline combined & $0.78_{-0.01}^{+0.01}$ & $1.43_{-0.07}^{+0.07}$ & $0.40_{-0.12}^{+0.15}$ & $1.15_{-0.17}^{+0.10}$ & $0.93_{-0.11}^{+0.12}$ & $0.86_{-0.20}^{+0.21}$ & $0.90_{-0.08}^{+0.09}$ & $7.7_{-0.7}^{+0.7}$ & $7.5_{-0.5}^{+0.5}$ & $795 / 423$ \\
\hline
\end{tabular}

${ }^{*}$ Normalization for the Mekal model, $N o r m=\int n_{\mathrm{e}} n_{\mathrm{H}} d V /\left(4 \pi(1+z)^{2} D_{\mathrm{A}}^{2}\right)$, where $D_{\mathrm{A}}$ is the angular distance to the source.

$2^{\prime} \leq r \leq 14^{\prime}$. Therefore, only the annulus of $2-4^{\prime}$ was simultaneously examined by both satellites. The spectra for $0.2^{\prime} \lesssim r \lesssim 1^{\prime}$ include two cavities. Since the temperature and abundance of the two cavities are consistent with that in the non-cavity region as shown previously $(\S 4.2)$, we did not exclude the cavity region and simply analyzed the annular spectra. Extraction of each spectrum and the treatment of the background are conducted in the same way as described in $\S 5.1$.

Spherical symmetry was assumed in the deprojection procedure. Starting from the outermost region, we fitted the projected annular spectrum with 1- $T$ or 2-T model. Spectrum 
Table 8. Same as table 7 , besides the element abundances are determined separately for the combined fit.

\begin{tabular}{|c|c|c|c|c|c|c|c|c|c|c|}
\hline & $\begin{array}{l}k T_{1} \\
(\mathrm{keV})\end{array}$ & $\begin{array}{l}k T_{2} \\
(\mathrm{keV})\end{array}$ & $\begin{array}{l}\text { O } \\
\text { (solar) }\end{array}$ & $\begin{array}{l}\mathrm{Mg} \\
\text { (solar) }\end{array}$ & $\begin{array}{l}\mathrm{Si} \\
\text { (solar) }\end{array}$ & $\begin{array}{l}\text { S } \\
\text { (solar) }\end{array}$ & $\begin{array}{l}\mathrm{Fe} \\
\text { (solar) }\end{array}$ & $\begin{array}{l}\text { Norm }_{1} \\
\quad\left(10^{-18}\right.\end{array}$ & $\begin{array}{l}\text { Norm }_{2} \\
\left.\mathrm{~cm}^{-5}\right)\end{array}$ & $\chi^{2} /$ dof \\
\hline vMekal & $0.71_{-0.01}^{+0.02}$ & $1.31_{-0.07}^{+0.14}$ & $0.37_{-0.10}^{+0.11}$ & $1.17_{-0.18}^{+0.21}$ & $1.06_{-0.14}^{+0.16}$ & $1.01_{-0.25}^{+0.28}$ & $0.86_{-0.08}^{+0.08}$ & $6.2_{-0.6}^{+0.8}$ & $8.3_{-1.3}^{+1.0}$ & $801 / 418$ \\
\hline \multirow[t]{2}{*}{ vAPEC } & $0.78_{-0.01}^{+0.01}$ & $1.44_{-0.09}^{+0.08}$ & $0.42_{-0.09}^{+0.11}$ & $1.13_{-0.17}^{+0.23}$ & $0.95_{-0.11}^{+0.16}$ & $0.98_{-0.22}^{+0.26}$ & $0.83_{-0.09}^{+0.11}$ & $8.1_{-1.2}^{+1.1}$ & $6.9_{-0.8}^{+0.7}$ & $783 / 418$ \\
\hline & & & $\mathrm{Ne}$ & $\mathrm{Al}$ & $\mathrm{Ar}$ & $\mathrm{Ca}$ & $\mathrm{Ni}$ & & & \\
\hline vMekal & & & $0.49_{-0.45}^{+0.50}$ & $2.70_{-1.32}^{+1.38}$ & $0.67_{-0.67}^{+1.02}$ & $0.88_{-0.88}^{+2.12}$ & $2.33_{-0.70}^{+0.77}$ & & & \\
\hline vAPEC & & & $0.72_{-0.29}^{+0.34}$ & $1.89_{-1.21}^{+1.41}$ & $0.72_{-0.72}^{+1.01}$ & $0.00_{-0.00}^{+1.73}$ & $1.80_{-0.69}^{+0.84}$ & & & \\
\hline
\end{tabular}

Table 9. Fractional contribution of the outer shells to the inner shells in the deprojection analysis.

\begin{tabular}{|c|c|c|c|c|c|}
\hline & $4-8^{\prime}$ & $2-4^{\prime}$ & $1-2^{\prime}$ & $0.6-1^{\prime}$ & $0-0.6^{\prime}$ \\
\hline $8-14^{\prime} \ldots$ & 0.373 & 0.075 & 0.018 & 0.004 & 0.002 \\
\hline $4-8^{\prime} \ldots \ldots$ & - & 0.273 & 0.056 & 0.012 & 0.007 \\
\hline $2-4^{\prime}$ & . & 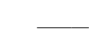 & 0.273 & 0.048 & 0.026 \\
\hline $1-2^{\prime} \ldots \ldots$ & 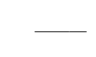 & 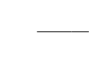 & - & 0.238 & 0.109 \\
\hline $0.6-1^{\prime} \ldots$ & - & - & - & - & 0.531 \\
\hline
\end{tabular}

of the neighboring inner region was then fitted with a model which contained contribution from the outer regions with fixed model parameters at their best-fit values. We repeated this procedure until the innermost region was reached. Because the outer contributions are fixed in the inner model fitting, the propagation of errors is ignored in this method. However, the inner regions are always brighter than outer regions, hence this effect is negligible. Table 9 summarizes the fractional contribution of the outer shells to the inner shells. For example, the spectrum of the $4-8^{\prime}$ annulus was fitted with adding $37.3 \%$ flux of the best-fit model in the 8-14' annulus.

Without the deprojection, the metal abundance of the group center $\left(r \lesssim 1^{\prime}\right)$ is underestimated by about 30\%, while the temperature does not change significantly. The sample spectra in the $2-4^{\prime}$ annulus fitted with the deprojected 2-T model are shown in figure $8(\mathrm{~b})$, and the fit results are summarized in table 10. Figures $9 \& 10$ show the result of the deprojection analysis, and the 1- $T$ results are quite similar to the previous ASCA result (Finoguenov \& Ponman 1999).

\subsection{Temperature profile}

The temperature profiles obtained by both the 1- $T$ and 2- $T$ models with the deprojection analysis are shown in figure 9. For the radius range $0.6<r<2.0^{\prime}$, the $2-T$ model gives significantly lower $\chi^{2}$ values $\left(\chi^{2}=78,68\right.$ for $0.6-1.0^{\prime}, 1.0-2.0^{\prime}$, respectively) than the $1-T$ model $\left(\chi^{2}=159,126\right)$. The significance was tested with the $F$-test, and the $2-T$ model was preferred with a significance higher than $5 \sigma$. Within $r<0.6^{\prime}$, the $2-T$ model is preferred for the whole 
Table 10. Results of the deprojected spectral fit with 1-T and 2-T vMekal models. The Chandra data (ACIS-S3) are used for $0-4^{\prime}$, and the XMM-Newton (MOS1+2, pn) data are used for $2-14^{\prime}$.

\begin{tabular}{|c|c|c|c|c|c|c|c|c|c|c|}
\hline $\begin{array}{l}r \\
(\operatorname{arcmin})\end{array}$ & $\begin{array}{l}k T_{1} \\
(\mathrm{keV})\end{array}$ & $\begin{array}{l}k T_{2} \\
(\mathrm{keV})\end{array}$ & $\begin{array}{l}\mathrm{O}, \mathrm{Ne} \\
(\text { solar })\end{array}$ & $\begin{array}{l}\mathrm{Mg}, \mathrm{Al} \\
\text { (solar) }\end{array}$ & $\begin{array}{l}\mathrm{Si} \\
\text { (solar) }\end{array}$ & $\begin{array}{l}\mathrm{S}, \mathrm{Ar}, \mathrm{Ca} \\
\text { (solar) }\end{array}$ & $\begin{array}{l}\mathrm{Fe}, \mathrm{Ni} \\
\text { (solar) }\end{array}$ & $\begin{array}{l}\operatorname{Norm}_{1}{ }^{*} \\
\quad\left(10^{-18}\right.\end{array}$ & $\begin{array}{l}\mathrm{Norm}_{2}{ }^{*} \\
\left.\mathrm{~cm}^{-5}\right)\end{array}$ & $\chi^{2} /$ dof \\
\hline \multicolumn{11}{|c|}{ 1- $T$ vMekal model } \\
\hline \multicolumn{11}{|c|}{ Chandra results } \\
\hline $0.0-0.6$ & $0.73 \pm 0.01$ & - & $0.45_{-0.14}^{+0.18}$ & $1.20_{-0.29}^{+0.39}$ & $1.10_{-0.26}^{+0.34}$ & $<0.49$ & $0.76_{-0.10}^{+0.16}$ & $4.6_{-1.2}^{+1.0}$ & - & $177 / 96$ \\
\hline $0.0-0.2$ & $0.67 \pm 0.02$ & - & $0.47_{-0.26}^{+0.49}$ & $1.30_{-0.60}^{+1.12}$ & $0.63_{-0.51}^{+0.75}$ & $<1.03$ & $0.74_{-0.20}^{+0.44}$ & $1.2_{-0.7}^{+0.6}$ & - & $75 / 57$ \\
\hline $0.2-0.4$ & $0.70_{-0.02}^{+0.03}$ & - & $0.37_{-0.26}^{+0.53}$ & $1.61_{-0.70}^{+1.52}$ & $1.53_{-0.73}^{+1.43}$ & $<1.47$ & $0.80_{-0.23}^{+0.58}$ & $1.2_{-0.8}^{+0.6}$ & - & $96 / 66$ \\
\hline $0.4-0.6$ & $0.77 \pm 0.03$ & - & $0.73_{-0.49}^{+1.17}$ & $1.28_{-0.97}^{+1.95}$ & $1.79_{-1.03}^{+2.14}$ & $1.53^{\dagger}$ & $1.13_{-0.37}^{+0.98}$ & $0.7 \pm 0.3$ & - & $67 / 67$ \\
\hline $0.6-1.0$ & $0.84 \pm 0.01$ & - & $0.27_{-0.17}^{+0.23}$ & $1.53_{-0.46}^{+0.65}$ & $1.52_{-0.40}^{+0.56}$ & $<0.67$ & $0.78_{-0.13}^{+0.21}$ & $2.7_{-0.5}^{+0.5}$ & - & $159 / 92$ \\
\hline $1.0-2.0$ & $1.12 \pm 0.01$ & - & $0.03_{-0.03}^{+0.13}$ & $0.71_{-0.28}^{+0.31}$ & $0.70_{-0.18}^{+0.20}$ & $0.49_{-0.47}^{+0.51}$ & $0.48_{-0.05}^{+0.06}$ & $6.2_{-0.7}^{+0.6}$ & - & $126 / 72$ \\
\hline $2.0-4.0$ & $1.37_{-0.04}^{+0.03}$ & - & $0.02_{-0.02}^{+0.25}$ & $0.35_{-0.35}^{+0.53}$ & $0.99_{-0.34}^{+0.40}$ & $0.55_{-0.55}^{+0.76}$ & $0.52_{-0.08}^{+0.10}$ & $5.6_{-1.0}^{+0.8}$ & - & $128 / 108$ \\
\hline \multicolumn{11}{|c|}{ XMM-Newton results } \\
\hline $2.0-4.0$ & $1.43_{-0.05}^{+0.17}$ & - & $<0.67$ & $0.47_{-0.47}^{+1.02}$ & $0.68_{-0.28}^{+0.34}$ & $0.54_{-0.47}^{+0.50}$ & $0.41_{-0.08}^{+0.18}$ & $5.6_{-1.2}^{+0.8}$ & - & $241 / 199$ \\
\hline $4.0-8.0$ & $1.41_{-0.07}^{+0.08}$ & - & $0.12_{-0.12}^{+0.37}$ & $0.58_{-0.58}^{+0.76}$ & $0.25_{-0.22}^{+0.24}$ & $<0.15$ & $0.26_{-0.06}^{+0.07}$ & $10.9_{-1.4}^{+1.3}$ & - & $164 / 156$ \\
\hline $8.0-14.0$ & $0.64 \pm 0.03$ & - & $0.24_{-0.07}^{+0.08}$ & $0.06_{-0.06}^{+0.10}$ & $0.08_{-0.08}^{+0.09}$ & $0.40_{-0.40}^{+0.53}$ & $0.04_{-0.01}^{+0.01}$ & $47.4_{-8.9}^{+9.8}$ & - & $188 / 180$ \\
\hline \multicolumn{11}{|c|}{ combined results } \\
\hline $2.0-4.0$ & $1.39 \pm 0.03$ & - & $<0.21$ & $0.40_{-0.40}^{+0.49}$ & $0.79_{-0.23}^{+0.27}$ & $0.57_{-0.42}^{+0.45}$ & $0.47_{-0.06}^{+0.08}$ & $5.6_{-0.6}^{+0.4}$ & - & $415 / 314$ \\
\hline \multicolumn{11}{|c|}{$2-T$ vMekal model } \\
\hline \multicolumn{11}{|c|}{ Chandra results } \\
\hline $0.0-0.6$ & $0.66_{-0.22}^{+0.03}$ & $0.92_{-0.47}^{+0.18}$ & $0.39_{-0.14}^{+0.24}$ & $1.21_{-0.31}^{+0.61}$ & $1.18_{-0.29}^{+0.54}$ & $<1.05$ & $0.84_{-0.12}^{+0.31}$ & $3.1_{-2.0}^{+0.7}$ & $1.4_{-0.7}^{+2.8}$ & $151 / 94$ \\
\hline $0.6-1.0$ & $0.77_{-0.07}^{+0.04}$ & $1.36_{-0.20}^{+0.47}$ & $0.42_{-0.27}^{+0.56}$ & $1.92_{-0.79}^{+1.45}$ & $2.25_{-0.74}^{+1.37}$ & $1.02_{-1.02}^{+2.09}$ & $1.40_{-0.39}^{+0.75}$ & $1.1_{-0.4}^{+0.5}$ & $1.0_{-0.3}^{+0.5}$ & $78 / 90$ \\
\hline $1.0-2.0$ & $0.83_{-0.15}^{+0.06}$ & $1.36_{-0.11}^{+0.19}$ & $0.29_{-0.24}^{+0.33}$ & $1.20_{-0.58}^{+0.76}$ & $1.08_{-0.39}^{+0.52}$ & $1.24_{-0.89}^{+1.14}$ & $0.99_{-0.22}^{+0.31}$ & $0.7_{-0.4}^{+0.5}$ & $2.8_{-1.0}^{+0.8}$ & $69 / 70$ \\
\hline $2.0-4.0$ & $0.85_{-0.23}^{+0.29}$ & $1.59_{-0.20}^{+0.69}$ & $0.14_{-0.14}^{+0.57}$ & $1.05_{-0.87}^{+1.25}$ & $2.02_{-0.74}^{+1.16}$ & $1.48_{-1.25}^{+1.71}$ & $1.11_{-0.33}^{+0.54}$ & $0.3_{-0.2}^{+0.9}$ & $3.2_{-0.9}^{+0.8}$ & $121 / 106$ \\
\hline \multicolumn{11}{|c|}{ XMM-Newton results } \\
\hline $2.0-4.0$ & $0.90_{-0.21}^{+0.27}$ & $1.83_{-0.22}^{+0.51}$ & $0.16_{-0.16}^{+1.24}$ & $2.13_{-1.86}^{+3.95}$ & $1.57_{-0.70}^{+1.68}$ & $1.07_{-0.84}^{+1.32}$ & $1.02_{-0.38}^{+1.06}$ & $0.3_{-0.2}^{+0.6}$ & $3.2_{-1.1}^{+1.0}$ & $235 / 197$ \\
\hline $4.0-8.0$ & $0.24_{-0.04}^{+0.08}$ & $1.40_{-0.06}^{+0.08}$ & $0.13_{-0.10}^{+0.19}$ & $1.04_{-0.71}^{+0.89}$ & $0.39_{-0.25}^{+0.31}$ & $<0.22$ & $0.32_{-0.09}^{+0.11}$ & $3.2_{-1.7}^{+3.3}$ & $10.4_{-1.7}^{+1.7}$ & $153 / 154$ \\
\hline $8.0-14.0$ & $0.43_{-0.09}^{+0.51}$ & $0.89_{-0.56}^{+0.13}$ & $0.19_{-0.08}^{+0.10}$ & $0.07_{-0.07}^{+0.17}$ & $0.10_{-0.10}^{+0.12}$ & $0.31_{-0.31}^{+0.52}$ & $0.06_{-0.02}^{+0.05}$ & $23.9_{-11.5}^{+21.5}$ & $22.9_{-11.5}^{+12.4}$ & $185 / 178$ \\
\hline \multicolumn{11}{|c|}{ combined results } \\
\hline $2.0-4.0$ & $0.89_{-0.12}^{+0.23}$ & $1.71_{-0.15}^{+0.48}$ & $0.13_{-0.13}^{+0.47}$ & $1.23_{-0.82}^{+1.10}$ & $1.68_{-0.52}^{+0.74}$ & $1.23_{-0.70}^{+0.88}$ & $1.05_{-0.26}^{+0.39}$ & $0.4_{-0.1}^{+0.6}$ & $3.3_{-0.7}^{+0.6}$ & $402 / 312$ \\
\hline
\end{tabular}

* Normalization for the vMekal model, Norm $=\int n_{\mathrm{e}} n_{\mathrm{H}} d V /\left(4 \pi(1+z)^{2} D_{\mathrm{A}}^{2}\right)$, where $D_{\mathrm{A}}$ is the angular distance to the source.

$\dagger$ The S abundance was fixed to the best fit in calculating errors due to an unresolved problem on XSPEC for this particular fit.

$0.0-0.6^{\prime}$ data by $\Delta \chi^{2}=26$ (99.9\% confidence with $F$-test), while the $1-T$ model is also acceptable when we split the region into smaller annuli, $0.0-0.2^{\prime}, 0.2-0.4^{\prime}$, and $0.4-0.6^{\prime}$. Furthermore, we have also tried the $2-T$ model for the spectra in the range $0-0.2^{\prime}$ and $0.2-0.4^{\prime}$, but the normalization of the second component turned out to be nil. This is due probably to the poorer statistics for the smaller annuli and also to the fact that the cooler component is dominant in the central region. In the outer regions of $r>2^{\prime}$, the $\chi^{2}$ values for the 2-T model were also lower 

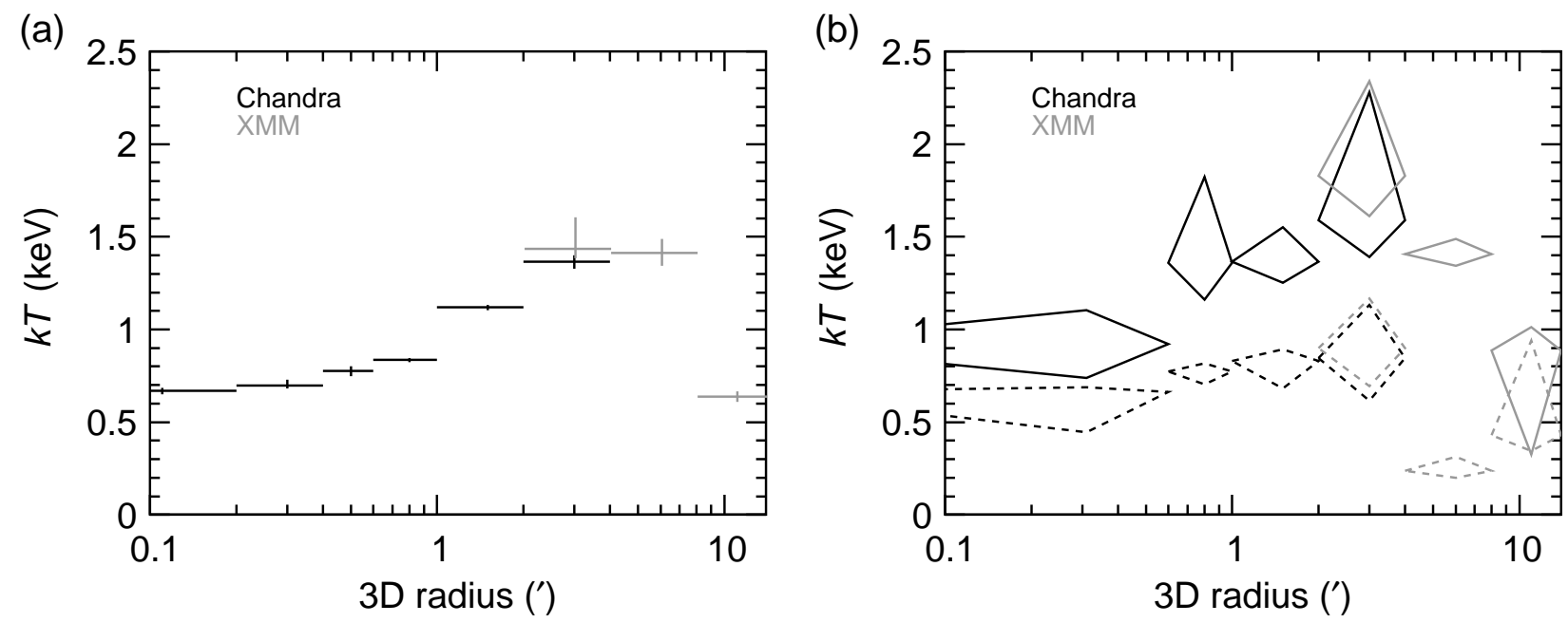

Fig. 9. (a) Temperature profiles with the 1-T vMekal models based on the deprojected spectral fit. ACIS-S3 (black) is used for $r<4^{\prime}$, and MOS1, MOS2, and pn (gray) are used for $2^{\prime}<r<14^{\prime}$. (b) Same as (a), but with the $2-T$ vMekal models.

than the 1- $T$ case, but both of the fit were acceptable. For the outermost range $8-14^{\prime}$, the $2-T$ model gave similar temperatures for the hotter and cooler components within errors, therefore the 1- $T$ fit turned out to be just enough. This is because the hot component dominates the surface brightness.

For the 1- $T$ model, the temperature rises from $k T=0.7 \mathrm{keV}$ at the center to $k T=1.4 \mathrm{keV}$ at larger radii, which is consistent with the previous deprojection analysis for the ASCA data (Finoguenov \& Ponman 1999) and the ROSAT temperature profile by Buote (2000a). This profile in $r<2^{\prime}$ is also seen in the temperature map calculated from the $H R$ (figure 6 ). The temperature with the 1- $T$ model drops again at $r>8^{\prime}$ from the intermediate level of $k T=$ $1.4 \mathrm{keV}$, as shown in figure 9. The temperature drop in our data is much steeper than those reported by Finoguenov \& Ponman (1999) with ASCA and by Buote (2000a) with ROSAT. The ROSAT temperature at $r>8^{\prime}$ is $k T=0.9-1.0 \mathrm{keV}$, while ours is $k T=0.66 \pm 0.03 \mathrm{keV}$. The influence of the background has been tested by changing the background level within $\pm 5 \%$, and we found that the best fit temperature varied in $0.64-0.70 \mathrm{keV}$. We have looked into the effects of point source contamination and the influence of hard and soft components, though none of them gives significant change in temperature.

For the 2- $T$ model, temperature of the cooler component is nearly constant at $\sim 0.7 \mathrm{keV}$, which is very close to the central temperature obtained with the $1-T$ fit. Temperature of the hotter component is also nearly constant at $\sim 1.4 \mathrm{keV}$ up to $r \sim 8^{\prime}$. These features are consistent with the ASCA results, which indicate two temperature components at $0.7 \mathrm{keV}$ and $1.4 \mathrm{keV}$ based on projected spectra within $r<3^{\prime}$ (Buote 2000b). The cool component is concentrated in the center and dominant within $1^{\prime}$, while the hot component extends out to $\sim 4^{\prime}$. The extent of the cool component will be examined in $\S 6$ more quantatively. 

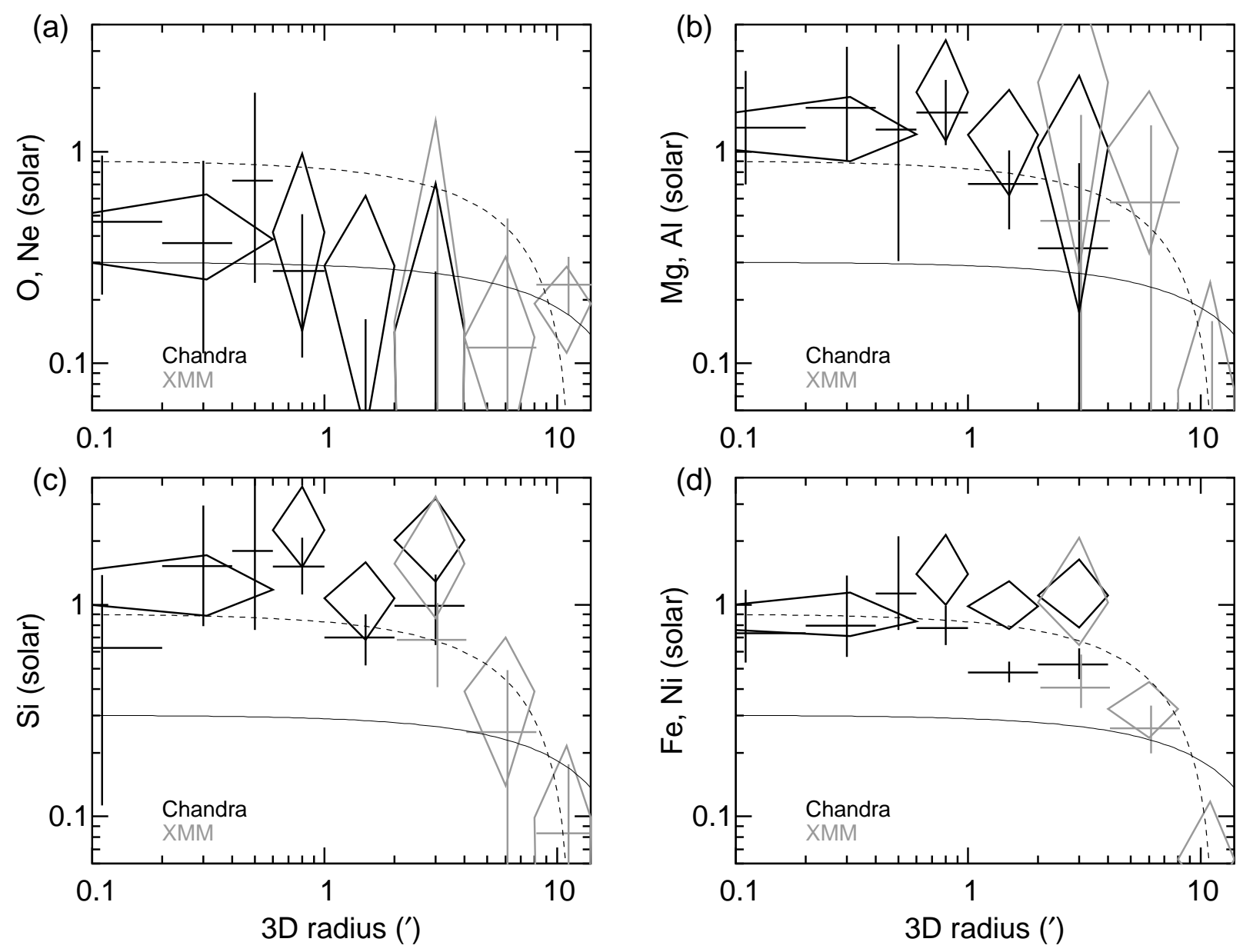

Fig. 10. Abundance profiles of $\mathrm{O}$ (with $\mathrm{Ne}$ ) (a), $\mathrm{Mg}(\mathrm{b}), \mathrm{Si}(\mathrm{c})$, and $\mathrm{Fe}$ (with $\mathrm{Ni}$ ) (d) using the 1-T (crosses) and 2-T (diamonds) vMekal models with the deprojection analysis. The ACIS-S3 (black) is used for $r<4^{\prime}$, and MOS1, MOS2, and pn (gray) for $2^{\prime}<r<14^{\prime}$. The solar photospheric value of $[\mathrm{Fe} / \mathrm{H}]=4.7 \times 10^{-5}$ (Anders and Grevesse 1989) is adopted for the Fe abundance. The solid and dashed lines correspond to the best fit relations of the deprojected $\mathrm{O}$ and Fe profile of the 2- $T$ results, respectively.

\subsection{Abundance profile}

The metal abundances were derived from the deprojected spectra based on the 1- $T$ and 2- $T$ fits. The $\mathrm{Fe}, \mathrm{Si}$, and $\mathrm{Mg}$ abundances are around one solar or more in $r<4^{\prime}$. On the other hand, the $\mathrm{O}$ abundance is always lower than the solar value (table 10 and figure 10). We could poorly constrain the $\mathrm{S}$ abundance due to the limited statistics. The $2-T$ fit gave significantly higher abundance than the $1-T$ fit in the intermediate range of $1.0^{\prime}<r<4.0^{\prime}$, while similar abundances between 1-T and 2- $T$ are obtained for the inner $\left(r<1.0^{\prime}\right)$ and outer $\left(4.0^{\prime}<r\right)$ regions. Such discrepancy is often seen in the spectral fit, because the abundance takes the lowest value at the temperature where the line emissivity is the highest. This situation corresponds to the case of 1- $T$ fit, and the 2- $T$ model tends to shift the temperature away from such a high emissivity position. The ASCA spectra of HGG 62 are fitted with the 1-T Mekal model by Finoguenov \& Ponman (1999), and the present results for $\mathrm{Fe}$, Si, and $\mathrm{Mg}$ with 1-T 
fits are in good agreement with the ASCA deprojection results within the $90 \%$ confidence limit at $r>1^{\prime}$,

In figure 10, the solid and dashed lines correspond to the best fit relations of the deprojected $\mathrm{O}(\mathrm{a})$ and $\mathrm{Fe}(\mathrm{d})$ profile of the 2-T results, respectively. A simple linear model of $Z=-a r+b$ is assumed for each profile. These best fit relations are overlaid to all the four plots for comparison. O abundance of $\sim 0.3$ solar is significantly lower than the other elements. It also indicates a flatter profile, though significance is low due to the low abundance of oxygen. The decline of $\mathrm{Fe}, \mathrm{Si}$, and $\mathrm{Mg}$ abundances with radius previously reported by Finoguenov \& Ponman (1999) with ASCA, is also confirmed with Chandra and XMM-Newton. Our result shows that $\mathrm{Fe}, \mathrm{Si}$, and $\mathrm{Mg}$ abundances are 1-2 solar at the center and drop to about 0.1 solar at $r \sim 10^{\prime}$. These abundance levels are about twice larger than the values derived with the ASCA spectra ( $\sim 0.6$ solar by Finoguenov \& Ponman 1999). This is due primarily to the much better angular resolution of the Chandra X-ray telescope $\left(0.5^{\prime \prime}\right)$ than ASCA $\left(3^{\prime}\right)$ at the very central region. In addition, our 2- $T$ model gives higher abundances in the intermediate range of $1.0^{\prime}<r<4.0^{\prime}$. As described in $\S 5.4$, the $2-T$ model is significantly needed in this region, so that the ASCA abundances are likely to be underestimated. We also note that many authors recently take the solar abundance to be the value given by Grevesse \& Sauval (1998), i.e. $[\mathrm{Fe} / \mathrm{H}]=3.2 \times 10^{-5}$, which is obtained from the measurements of solar system meteorites. The solar photospheric value of $[\mathrm{Fe} / \mathrm{H}]=4.7 \times 10^{-5}$ (Anders and Grevesse 1989), which is adopted in our analysis, gives the Fe abundance approximately by a factor of 1.47 smaller. Considering this effect, all the $\mathrm{Fe}$, $\mathrm{Si}$, and $\mathrm{Mg}$ abundances have similar value around 1.2 solar at the center $\left(r<0.6^{\prime}\right)$.

\section{Mass Profiles}

\subsection{Formulation of two-phase gas}

Based on the radial distributions of the cool and hot components, we will derive mass distributions of gas and dark matter. Since the estimated gas mass contained in a single cavity volume, if it is filled up, is $\sim 6 \%$ of the mass in the shell of $7^{\prime \prime}<r<32^{\prime \prime}$, the assumption of the spherical symmetry gives relatively small errors. However, because two temperatures are required for each shell within the deprojected radius of $0.6^{\prime}-4.0^{\prime}$ as seen in the previous section, we need an additional assumption between the hot and cool components. Here we assume a pressure balance between the two phase, i.e. $P_{\text {gas }}=n_{1} k T_{1}=n_{2} k T_{2}$, where $n_{1}, n_{2}, T_{1}$ and $T_{2}$ are the cool and hot gas densities and temperatures, respectively, at each 3 -dimensional radius of $R$. Such a pressure balance is previously adopted by Ikebe et al. (1999) for the Centaurus cluster, and by Xue et al. (2004) for the RGH 80 galaxy group.

We also introduce a volume filling factor, $f(R)$, of the cool component, namely a fractional volume, $V_{1} \equiv f V$, in the total volume, $V$, is filled with the cool gas, and $V_{2} \equiv(1-f) V$ 
is filled with the hot gas. This means that the cool and hot gas are not completely mixed, instead the cool component is somewhat patchy or localized with rather an irregular shape. Such a hypothesis may be supported by the 2-dimensional temperature map (figure 6) and the existence of the cavities.

Because the normalizations of the 2- $T$ vMekal models are expressed as $N_{o r m}=C_{12} n_{1}^{2} V_{1}$ and Norm $_{2}=C_{12} n_{2}^{2} V_{2}$ using a certain common constant, $C_{12}$, the volume filling factor, $f(R)$, can be solved under the pressure balance as,

$$
f(R)=\left[1+\left(\frac{T_{2}}{T_{1}}\right)^{2} \frac{\text { Norm }_{2}}{\text { Norm }_{1}}\right]^{-1} .
$$

We have calculated this formula for each shell in $0.6-1.0^{\prime}, 1.0-2.0^{\prime}, 2.0-4.0^{\prime}$, and $4.0-8.0^{\prime}$ using the best-fit values in table 10 to plot $f(R)$ against the 3-dimensional radius $R$ in figure 11 (a). This plot clearly indicates that the cool component is dominant at the central region, while it occupies only $\lesssim 1 \%$ at $R>4^{\prime}$. We have fitted these four points with a 3 -dimensional $\beta$-model function, $f(R)=\left[1+\left(R / R_{\mathrm{c}, f}\right)^{2}\right]^{-3 \beta_{f} / 2}$, and obtained $R_{\mathrm{c}, f}=0.43_{-0.16}^{+0.19}$ and $\beta_{f}=0.60_{-0.13}^{+0.19}$.

Considering the projection effect, we can calculate the volume occupied by the cool gas at each 2-dimensional ring of $\theta_{\text {in }}-\theta_{\text {out }}$ in unit of radian, as

$$
V_{1}=f \frac{4}{3} \pi D_{\mathrm{A}}^{3}\left(\theta_{\mathrm{out}}^{3}-\theta_{\mathrm{in}}^{3}\right)\left(1-\theta_{\mathrm{r}}^{2}\right)^{3 / 2} /\left(1-\theta_{\mathrm{r}}^{3}\right),
$$

where $\theta_{\mathrm{r}} \equiv \theta_{\text {in }} / \theta_{\text {out }}$, and $D_{\mathrm{A}}=61 \mathrm{Mpc}$ is the angular diameter distance to HCG 62. Because the vMekal normalization is defined as $N o r m=\int n_{\mathrm{e}} n_{\mathrm{H}} d V /\left(4 \pi(1+z)^{2} D_{\mathrm{A}}^{2}\right)$, the electron density of the cool gas, $n_{\mathrm{e} 1}$, is computed as

$$
n_{\mathrm{e} 1}=\sqrt{1.2 \times 4 \pi(1+z)^{2} D_{\mathrm{A}}^{2} / V_{1}},
$$

assuming $n_{\mathrm{e}}=1.2 n_{\mathrm{H}}$ for a fully ionized gas with hydrogen and helium mass fraction of $X=0.7$ and $Y=0.28$. The electron density of the hot gas, $n_{\mathrm{e} 2}$, is similarly derived by replacing $f$ into $(1-f)$. The ion density including helium is $n_{\mathrm{i}}=0.92 n_{\mathrm{e}}$, therefore the gas pressure is calculated as

$$
P_{\text {gas }}=1.92 n_{\mathrm{e} 1} k T_{1}=1.92 n_{\mathrm{e} 2} k T_{2} .
$$

The derived gas pressure $P_{\text {gas }}$, cool or hot gas temperature $k T_{1}$ or $k T_{2}$, and cool or hot electron density $n_{\mathrm{e} 1}$ or $n_{\mathrm{e} 2}$ are plotted in figures $11(\mathrm{~b})-(\mathrm{d})$. The 2- $T$ fit results are adopted for points in the range of $0.6-4^{\prime}$ and the 1- $T$ fit results for others. The systematic errors when the background level is increased or decreased by $\pm 5 \%$ are considered in the error bars for the outermost two points. It is supposed that blue points belongs to the cool component and red points to the hot one.

We must be careful in dealing with these plots because data points are not independent with each other. We first fitted the gas pressure plot by combining both the cool and hot data points, with a 3 -dimensional double $\beta$-model, $P_{\text {gas }}=P_{1}\left[1+\left(R / R_{\mathrm{c}, P_{1}}\right)^{2}\right]^{-3 \beta_{P_{1}} / 2}+$ $P_{2}\left[1+\left(R / R_{\mathrm{c}, P_{2}}\right)^{2}\right]^{-3 \beta_{P_{2}} / 2}$. Due to the small number of data points, we have fixed $R_{\mathrm{c}, P_{1}}=0.1^{\prime}$ and 
$\beta_{P_{1}}=0.65$ using the best-fit values in table 3 obtained with the radial surface brightness profile fit. The fitted parameters are $P_{1}=58 \pm 130 \mathrm{eV} \mathrm{cm}^{-3}, P_{2}=18 \pm 32 \mathrm{eV} \mathrm{cm}^{-3}, R_{\mathrm{c}, P_{2}}=0.60 \pm 1.44^{\prime}$, and $\beta_{P_{2}}=0.38 \pm 0.23$. The best-fit model is indicated by a dashed line in figure 11 (c). The green curves represent each component of the double $\beta$-model.

We then fitted the cool gas temperature with a power-law model, $k T_{1}(R)=a R^{b}$, and the hot gas electron density with a 3 -dimensional $\beta$-model and a constant, $n_{\mathrm{e} 2}(R)=$ $n_{\mathrm{e} 2,0}\left[1+\left(R / R_{\mathrm{c}, n_{\mathrm{e} 2}}\right)^{2}\right]^{-3 \beta_{n_{\mathrm{e} 2}} / 2}+C_{n_{\mathrm{e} 2}}$. The best-fit values are $a=0.80 \pm 0.17 \mathrm{keV}, b=0.09 \pm 0.20$, $n_{\mathrm{e} 2,0}=0.007 \pm 0.020 \mathrm{~cm}^{-3}, R_{\mathrm{c}, n_{\mathrm{e} 2}}=1.4 \pm 7.2^{\prime}, \beta_{n_{\mathrm{e} 2}}=1.2 \pm 5.8$, and $C_{n_{\mathrm{e} 2}}=(0.49 \pm 0.48) \times$ $10^{-3} \mathrm{~cm}^{-3}$. The best-fit models for $k T_{1}$ and $n_{\mathrm{e} 2}$ are drawn by dashed lines. The model functions for $k T_{2}$ and $n_{\mathrm{e} 1}$ are derived using the relation $k T_{2}=P_{\text {gas }} / n_{\mathrm{e} 2}$ and $n_{\mathrm{e} 1}=P_{\text {gas }} /\left(k T_{2}\right)$, which are indicated by another dashed line in each panel. Although errors of these parameters are quite large, these functions can reproduce the observed properties of the cool and hot gases fairly well.

\subsection{Dark matter and gas mass}

The gas mass density $\rho_{\text {gas }}$ is expressed as

$$
\rho_{\text {gas }}=1.92 \mu m_{\mathrm{p}}\left[f n_{\mathrm{e} 1}+(1-f) n_{\mathrm{e} 2}\right],
$$

where $\mu=0.62$ is the mean molecular weight, and $m_{\mathrm{p}}$ is the proton mass. Assuming the hydrostatic equilibrium, the total integrated gravitational mass, $M_{<R}$, within the 3-dimensional radius of $R$ is given by

$$
M_{<R}=-\frac{R^{2}}{\rho_{\text {gas }} G} \frac{d P_{\text {gas }}}{d R},
$$

in which $G$ is the gravitational constant. The differential mass density, $M(R)$, is given by

$$
M(R)=\frac{1}{4 \pi R^{2}} \frac{d M_{<R}}{d R} .
$$

Figures 11 (e) and (f) show the integrated and differential mass profiles (black lines) as a function of the 3-dimensional radius in unit of arcmin or kpc. We also overlaid the gas mass (gray) for the hot (red) and cool (blue) components in the same panels, by integrating the gas density, $\rho_{\text {gas }}$.

However, in the two-phase model under the pressure balance, the hot gas is lighter in mass density than the cool gas, and becomes buoyant. The hot gas would escape from the group core if it is not enclosed by other mechanisms (see $\S 8$ in detail). In such a case, hydrostatic equilibrium may have been broken, hence we have also calculated the integrated gravitational mass by treating the hot and cool components separately. Namely,

$$
\begin{aligned}
M_{1,<R} & =-\frac{R^{2}}{\rho_{1} G} \frac{d P_{\mathrm{gas}}}{d R}, \quad \rho_{1}=1.92 \mu m_{\mathrm{p}} n_{\mathrm{e} 1}, \\
M_{2,<R} & =-\frac{R^{2}}{\rho_{2} G} \frac{d P_{\mathrm{gas}}}{d R}, \quad \rho_{2}=1.92 \mu m_{\mathrm{p}} n_{\mathrm{e} 2}, \\
M_{<R} & =f M_{1,<R}+(1-f) M_{2,<R} .
\end{aligned}
$$



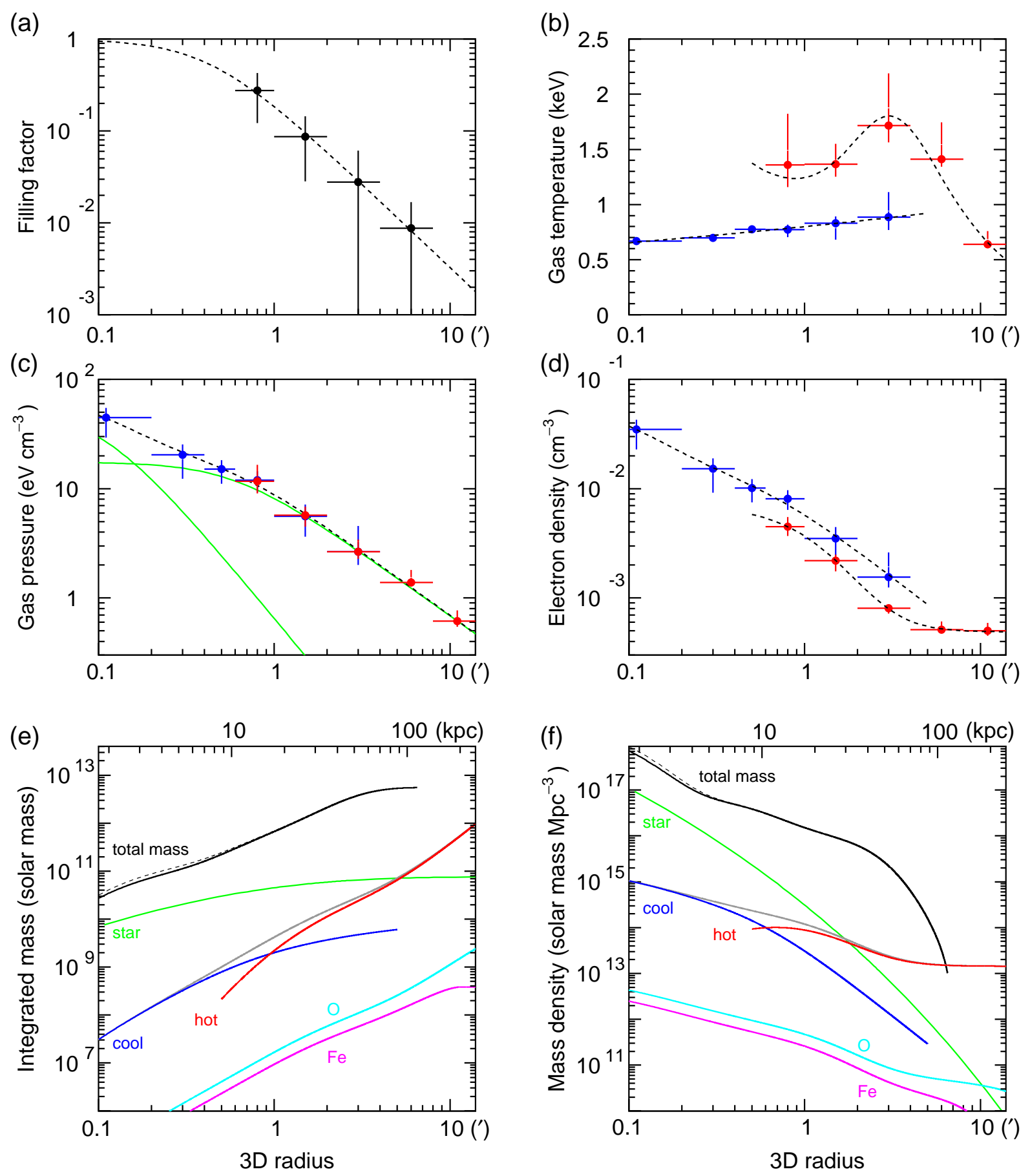

Fig. 11. (a) Volume filling factor $f(R)$ of the cool component, (b) cool or hot gas temperature $k T_{1}$ or $k T_{2}$, and (c) gas pressure $P_{\text {gas }}$, (d) cool or hot electron density $n_{\mathrm{e} 1}$ or $n_{\mathrm{e} 2}$, plotted against the 3-dimensional radius $R$. Blue points represent the cool component and red points the hot. In panels (b)-(d), 2-T fit results are adopted in the range of $0.6-4^{\prime}$ and $1-T$ fit results for others. The systematic errors when the background level is increased or decreased by $\pm 5 \%$ are considered in the error bars for the outermost two points. The dashed lines in panels (a)-(d) and green curves in panel (b) represent the best fit models. See text in details. (e) A black line indicates the total integrated gravitational mass, $M_{<R}$, estimated from the best fit models. The solid line is calculated by eq. (7), and dashed line is by eq. (9). Red and blue lines represent the gas mass of the hot and cool components, respectively. The $\mathrm{O}$ and $\mathrm{Fe}$ mass contained in either of the gas is plotted by cyan and pink lines. Th9stellar mass estimated from the $R$ band photometry of the HCG 62a galaxy by Tran et al. (2001) is indicated by a green line. See text for details. (f) Same as (e), but for the differential mass density profiles. 
The gravitational mass indicated by dashed lines in figures 11 (e) and (f) are calculated using eq. (9). There are only small differences between the two at the group center; the latter is larger by about $15 \%$ at $R=0.1^{\prime}(1.8 \mathrm{kpc})$.

We have encountered one severe problem in these plots. The integrated total mass saturates at $R \sim 6^{\prime}(100 \mathrm{kpc})$, and the differential mass becomes even smaller than the gas mass. This is physically unrealistic, suggesting that some presumption(s) we have supposed might not be realized around this radius. The direct source of the problem is caused by the fact that the hot gas temperature, $k T_{2}$, drops very steeply from $\sim 1.5 \mathrm{keV}$ to $0.64 \mathrm{keV}$ in this radial range. Therefore, the electron density, $n_{\mathrm{e}}$, almost saturates, while the gas pressure, $P_{\text {gas }}$, decreases monotonously. This might in part be affected by the smaller field of view in our observations than the extended group emission. We cannot deproject the outermost shell in 8-14', so that X-ray flux in this shell is slightly overestimated. Nevertheless, the major origin of the problem is due to the steep temperature drop. It should be examined more precisely by the Suzaku satellite, which has lower and more stable background than XMM-Newton, and much superior low-energy sensitivity than ASCA.

Another possibility, which is astrophysically more interesting, is that the hydrostatic equilibrium is not reached there. The flattening of the electron density suggests an outflow of the hot gas. The steep temperature drop can be reversely recognized as the temperature rise in the boundary region between the cool and hot gas. This suggests a shock heating of accreting ICM, and/or remnants of past lifted cavities (see $\S 8$ ). The apparent inconsistency in the total mass may therefore indicate traces of the dynamical evolution.

Aside from the above problem, the derived gravitational mass is about $M_{<R}=5 \times 10^{12} M_{\odot}$ within $100 \mathrm{kpc}$, close to the value given by Pildis et al. (1995), $M_{\text {tot }}=2.9 \times 10^{13} M_{\odot}$ within $r<15^{\prime}$ (270 kpc) with the ROSAT PSPC observation assuming a single-phase gas model. We also plot the $\mathrm{O}$ (cyan) and $\mathrm{Fe}$ (pink) mass contained in the whole gas. The simple linear fit plotted in figure 10 is adopted to calculate the $\mathrm{O}$ and $\mathrm{Fe}$ mass. The derived gas and Fe masses of $M_{\text {gas }<R}=1.6 \times 10^{12} M_{\odot}$ and $M_{\mathrm{Fe}<R}=4 \times 10^{8} M_{\odot}$ within $r<300 \mathrm{kpc}$ are consistent with the result from ASCA and ROSAT by Finoguenov \& Ponman $(1999)$ of $M_{\text {gas }<R}=(1.7 \pm 0.1) \times 10^{12} M_{\odot}$ and $M_{\mathrm{Fe}<R}=(3.8 \pm 2.0) \times 10^{8} M_{\odot}$. However, our values are based on the extrapolation out to the XMM-Newton field of view, so that they have larger errors by a factor of $\sim 2$.

\subsection{Stellar mass}

The stellar mass in figures 11 (e) and (f) indicated by green lines is estimated from the $R$ band photometry of the HCG 62a galaxy by Tran et al. (2001), assuming the spherical symmetry and the surface brightness profile obeying the de Vaucouleurs $r^{1 / 4}$ law,

$$
\Sigma(r) \propto 10^{-3.33071\left[\left(r / r_{\mathrm{eff}}\right)^{1 / 4}-1\right]},
$$

where $r_{\text {eff }}=32^{\prime \prime}$ is the effective radius, or the half light radius. Because the $r^{1 / 4}$ law is for the surface brightness, deprojection for the 3-dimensional radius must be performed. We utilized a 
numerical table computed by Young (1976). The mass-to-light ratio, $M_{\text {star }} / L_{B}=8\left(M_{\odot} / L_{B, \odot}\right)$, is assumed, and we use $B-R=2.0 \mathrm{mag}$ by Hickson et al. (1989), and $A_{B}=0.224$ and $A_{R}=0.139$ after NASA/IPAC Extragalactic Database (NED) to calculate the $B$ band absolute magnitude, $L_{B}=L_{R}+(B-R)-A_{B}+A_{R}=-19.46 \mathrm{mag}$. Using the value $L_{B, \odot}=5.48 \mathrm{mag}$, the stellar mass of the HCG 62 a galaxy is calculated as $M_{\text {star }}=7.6 \times 10^{10} M_{\odot}$.

The $B$ magnitude within the effective diameter of $59.90^{\prime \prime}$ isophotes (table 1 ) is also given by Hickson et al. (1989), however, we found that $M_{\text {star }}$ calculated from this value was by about a factor of four larger than the value calculated above, if we assume that $r_{\text {eff }}$ is the same for both bands. Then, the stellar mass would significantly exceed the total gravitational mass at the central region. We therefore have adopted the stellar mass based on the $R$ band photometry. Tran et al. (2001) also report that there is $19 \%$ of disk component which can be fitted by $\Sigma(r) \propto \exp \left(-r / r_{\mathrm{d}}\right)$ for HCG $62 \mathrm{a}$. This gives asymmetric image residual flux $R_{A}=19 \%$, and the total residual fraction of light $R_{T}=18 \%$. Therefore, uncertainty on the stellar mass estimation is at least $\sim 20 \%$.

We notice several features in the mass profiles. The gas mass of the cool component is taken over by the hot component at $\sim 1^{\prime}(18 \mathrm{kpc})$. The stellar mass is overcome by the gas mass at $\sim 2.5^{\prime}(45 \mathrm{kpc})$, indicating that the cool component is very concentrated in the group center. The region of high metal abundance almost coincides with the volume dominated by the stellar mass, which supports the natural view that stars are responsible for the production of excess iron and silicon around the group center.

\section{ICM properties}

The temperature structure in HCG 62 can be characterized by a mixture of hot $(\sim 1.4$ $\mathrm{keV})$ and cool $(\sim 0.7 \mathrm{keV})$ components. As shown in figure 11, the cool component is dominant within $r<0.8^{\prime}$ and then a cool and hot mixture appears in $r=0.8-4^{\prime}$. The hot component is dominant in the outer region. The two-phase nature seems to be preferred than the singlephase structure such as that seen in M 87 (Matsushita et al. 2002). However, this apparent two-phase does not necessarily mean that the cool and hot gas co-exist at the intermediate region, instead it probably represents that they are patchy and/or have irregular shapes. As seen in figure 6 , the cool region is elongated from northeast to southwest, roughly corresponding to the direction of the two cavities. In this sense, HCG 62 group is different from NGC 1399, NGC 5044 and RGH 80 galaxy groups (Buote 2002; Buote et al. 2003a; Xue et al. 2004), in which strong evidence for a multi-phase gas is suggested at the central region. On the other hand, $T_{\text {cool }}$ is similar to these groups, and $T_{\text {cool }} \simeq T_{\text {hot }} / 2$ is recognized among them. It is claimed that the temperatures of the cool component seen in these three groups are close to the kinetic temperature of the stars, although the stellar velocity dispersion is not known for the HCG 62a galaxy.

Mass density of the cool component exceeds the hot-component density within about 
$10 \mathrm{kpc}$, in which the stellar mass density is orders of magnitude higher. This again implies that the cool component is probably connected with the stars concentrated around the central galaxy HCG 62a. We also note that the cool component mass density is always smaller than the stellar mass density. On the other hand, the hot component exceeds the stellar mass density around $r \sim 2^{\prime}(30 \mathrm{kpc})$, indicating that the gravitational potential of the galaxy group is traced by the hot component. Its temperature $(1.4 \mathrm{keV})$ is also typical for groups of galaxies.

In the outer region $\left(r>8^{\prime}\right)$, the temperature of the hot component drops fairly sharply. The radius corresponds to about $0.15 r_{\text {vir }}$, with $r_{\text {vir }} \approx 1.1(T / 1.5 \mathrm{keV})^{1 / 2} \mathrm{Mpc}$. This causes a peak in the temperature profile at $r \approx 5^{\prime}(90 \mathrm{kpc})$. Similar temperature profiles have been observed in other galaxy groups such as RGH 80, NGC 2563, and NGC 5044 (Xue et al. 2004, Mushotzky et al. 2003, Buote et al. 2003a). The drop of temperature suggests either that the dark matter is confined within this small radius or that the gas is yet to be heated in this region. In either case, there is a certain boundary of the group around $0.2 r_{\text {vir }}$, and it is suggested that these groups are young and forming systems.

The heavy elements are enriched by SN Ia and SN II (Tsujimoto et al. 1995). The former process dominantly yields Fe group (Iwamoto et al. 1999), and the latter produces lighter elements like O, Ne and Mg (Thielemann et al. 1996; Nomoto et al. 2006). Both supernova contribute to $\mathrm{Si}$ and $\mathrm{S}$. Since $\mathrm{O}$ is produced mainly by SN II, the flatter O abundance profile compared with those of $\mathrm{Si}$ and Fe implies enhanced contribution of SN Ia in the central region and/or that the shallow potential of HCG 62 is unable to confine SN II products which should have been supplied to the intracluster space in the form of galactic winds. The products of SN Ia, on the other hand, are considered to be brought in to ICM by gas stripping.

The ratio, $\mathrm{Mg} / \mathrm{O}$, is $3.3 \pm 2.2$ times solar within $1^{\prime}$ (18 kpc) based on the $2-T$ fit. Though the error is large, this value is similar or somewhat larger than those in other groups: $2.5 \pm 0.4$ solar for NGC 5044 (Xu et al. 2002), $1.3 \pm 0.2$ for NGC 4636 (Tamura et al. 2003), both measured with RGS, and $\sim 2$ solar in RGH 80 (Xue et al. 2004). In M 87, this ratio is $1.3 \pm 0.1$ solar within $20 \mathrm{kpc}$ (Matsushita et al. 2003). Since both $\mathrm{O}$ and $\mathrm{Mg}$ are mainly produced by SN II, the marginally high $\mathrm{Mg} / \mathrm{O}$ value may reflect the difference in the stellar initial mass function (IMF). Theoretical calculations (Nomoto et al. 2006; Thielemann et al. 1996) predict that a lighter-mass progenitor of SN II synthesizes enhanced $\mathrm{Mg}$ compared with $\mathrm{O}$, therefore HCG 62 might have had the stellar IMF with smaller number of massive stars $\left(M \gtrsim 20 M_{\odot}\right)$ than our galaxy and other groups above. However, the dependence of the $\mathrm{Mg} / \mathrm{O}$ ratio on the progenitor mass is typically $\sim 20 \%$ at most, so that we have to think about other possibilities for the $\mathrm{Mg} / \mathrm{O}$ ratio greater than $\sim 1.2$.

If excess $\mathrm{Fe}$ in the center is caused by SN Ia activity of HCG 62a, it should be more extended at least than the extent of the central cool component which is the gas directly bound by the central galaxy. The possible enhancement of the $\mathrm{Ni} / \mathrm{Fe}$ ratio at $r<2^{\prime}$ as seen in $\S 5.1$ supports this scenario. For example, the CDD2 model in Iwamoto et al. (1999) predicts 
the $\mathrm{Ni} / \mathrm{Fe}$ ratio of 1.8 solar. The region of high metal abundance almost covers the volume dominated by the stellar mass, which supports the natural view that stars are responsible for the production of excess $\mathrm{Fe}$ and $\mathrm{Si}$ around the group center. It seems to range even to the 3D radius of $\sim 6^{\prime}(100 \mathrm{kpc})$ in figure 10 , where the hot gas mass exceeds the stellar mass as seen in figure 11 (f). This suggests either that the Fe production has occurred in a wider region than the present location of the galaxy or that there have been an outflow of Fe. The presence of cavities in HCG 62 suggests that part of the central metal-rich gas may have been lifted from the galaxy.

The observed abundance gradients indicate that there have been no strong mixing occurred in the core region. We also note that there is no significant change of temperature or abundance across the cavity regions. These features imply that the process of cavity creation causes mild, subsonic gas motion occurring in a fairly limited volume. This implication is consistent with the result by Brüggen \& Kaiser (2002) who showed that mixing by buoyant bubbles gave relatively weak impact on the metallicity gradients based on numerical simulations.

\section{Supporting mechanisms of X-ray cavities}

B04 have systematically studied 16 clusters, 1 group (HCG 62), and 1 galaxy (M84), in which prominent X-ray surface brightness depressions (cavities or bubbles) are observed. They find that a mechanical (kinetic) luminosity seems to correlate with the $1.4 \mathrm{GHz}$ synchrotron luminosity. However, its ratio ranges widely between a few and several thousand, and they have concluded that the radio luminosity is an unreliable gauge of the mechanical power of the AGN jets. Dunn \& Fabian (2004) and D05 have studied 21 clusters, 3 galaxies, in which HCG 62 is not included, and find that the ratio of an energy factor, $\mathcal{K}$, to a volume filling factor, $\mathcal{F}$, shows a large scatter, $1 \lesssim \mathcal{K} / \mathcal{F} \lesssim 1000$, for active cavities associated with radio lobes, and that it becomes even larger for ghost cavities. The factor, $\mathcal{K}$, accounts for the additional energy from relativistic particles accompanying the electrons (e.g., protons). The parameter of $\mathcal{F}$ represents the volume filling factor of the relativistic particles, and is supposed not to vary far from unity. Typical value of $\mathcal{K}$ in literature is $\mathcal{K}=100$, according to the measurements of cosmic-rays around the solar system. However, there is no direct evidence indicating that such a high energy density is really carried by protons. The reason why $\mathcal{K} / \mathcal{F}$ varies so largely from cluster to cluster is a mystery, which might imply that there are several ways in supporting mechanism and/or formation of the X-ray cavities.

In this section, we examine whether this non-thermal pressure support scenario is realistic or not for HCG 62. In $\S 8.1$, we summarize our X-ray results, as well as optical and radio observations. In $\S 8.2-8.4$, standard indices of cavities are calculated for $\mathrm{HCG} 62$, and difficulties in the non-thermal support scenario is considered in $\S 8.5$. In $\S 8.6$ and $\S 8.7$, other possibilities of the supporting mechanism and formation of cavities are investigated. 


\subsection{Characteristics in $X$-ray, optical, and radio}

We have confirmed the X-ray cavities reported by Vrtilek et al. $(2001,2002)$ in the northeast and southwest regions of HCG 62 in the Chandra image as shown in figures 3 (a) and 5 . As described in $\S 4.2$, absorption is unlikely to be the origin of cavities. There are no significant spectral differences between cavity and non-cavity regions, nor any traces of the shock-heated gas around the cavities, as seen in the temperature map (figure 6). According to our hollow sphere model analysis $(\S 4.3)$, both the cavities should be aligned side by side with the group core to a fairly good degree, namely, the projected distances to the group core are close to their real ones. It is also suggested that the shape of cavities is probably elongated in the direction of our line of sight, and that the side of the cavities which is farther from the core should be larger in size and/or weaker in the X-ray emissivity.

With regard to the central HCG 62a galaxy, we found no evidence for the AGN activity in the X-ray data. The best-fit spectrum of HCG $62 \mathrm{a}$ is a thermal emission rather than a power-law one. No point sources were recognized at the center of HCG 62a in both soft and hard bands. The upper limit of the AGN emission can be placed around $L_{\mathrm{X}} \lesssim 10^{39} \mathrm{erg} \mathrm{s}^{-1}$ $(0.5-4 \mathrm{keV})$. We also found that the location of HCG $62 \mathrm{a}$ was slightly shifted from the group core by about $5^{\prime \prime}(1.5 \mathrm{kpc})$ on the projected sky image (table 4$)$. This positional shift may imply the effect of gas stripping and/or the dynamical motion of the galaxy.

The ASCA has detected a spatially extended $\left(\sim 10^{\prime}\right)$ excess hard X-ray emission above $\sim 4 \mathrm{keV}$ in the HCG 62 group (Fukazawa et al. 2001), which is supposed to be due to the relativistic electrons with Lorentz factor $\gamma \sim 10^{3}-10^{4}$ and/or sub-relativistic particles. This may also have some relation to the X-ray cavity, however, we could not confirm nor reject this result due to the higher non X-ray background of Chandra and XMM-Newton than ASCA.

In the optical band, Coziol et al. (1998) and Shimada et al. (2000) have detected weak [NII] and [OI] emission lines in the spectrum of HCG 62a, and classified it as a low luminosity AGN (LLAGN). On the other hand, Coziol et al. (2004) have assigned the lowest activity index of -5 (quiescent, intermediate and old stellar populations) to HCG 62a, according to the equivalent width measurement of the $\mathrm{H} \alpha$ absorption lines. They have also distinguished HCG 62 group among 27 compact groups of galaxies as type C, which comprises groups with high velocity dispersions and are dominated by elliptical galaxies with no activity, presumably corresponding to the later stage of the evolution.

In the radio band, as shown in figure 3 (a), weak radio emission at $1.4 \mathrm{GHz}$ is detected around HCG 62a, however its luminosity is as small as $L_{\text {radio }}=1.8 \times 10^{38} \mathrm{erg} \mathrm{s}^{-1}(10 \mathrm{MHz}-5$ $\mathrm{GHz}$ ), smallest in table 1 of B04. The radio emission does not show a clear association with the cavities, though the angular resolution of $45^{\prime \prime} \mathrm{FWHM}$ is not sufficient to see detailed structures. 


\subsection{Gas pressure \& non-thermal pressure}

We take the distances to both cavities from the group core to be $R_{\text {cav }}=25^{\prime \prime}(7.4 \mathrm{kpc})$ and their radii to be $r_{\text {cav }}=13.5^{\prime \prime}(4.0 \mathrm{kpc})$ for simplicity. This approximation can be justified by the similarity between north and south cavities measured from the group core (table 5) with the center position determined from the center of the wider component in 2-dimensional 2- $\beta$ fit (table 4). Assuming those parameters, the ICM gas pressure at $R_{\text {cav }}$ is calculated to be $P_{\text {gas }}=17 \mathrm{eV} \mathrm{cm}^{-3}$, from eq. (5).

The non-thermal pressure of relativistic particles can be estimated from the radio intensity. Here, we assume that half of the radio flux comes from a single cavity, namely, $L_{\text {radio }}=9 \times 10^{37} \mathrm{erg} \mathrm{s}^{-1}(10 \mathrm{MHz}-5 \mathrm{GHz})$ according to B04. Assuming that the radio emission is due to synchrotron radiation by relativistic electrons, the total non-thermal pressure can be calculated as $P_{\text {tot }}=\mathcal{K} P_{\mathrm{e}}+\mathcal{F} P_{\mathrm{B}}$, where $P_{\mathrm{e}}$ is a pressure of the relativistic electrons, and $P_{\mathrm{B}}$ is a pressure of the magnetic field, following the convention adopted by D05.

The relativistic electron pressure is calculated to be $P_{\mathrm{e}}=C_{\mathrm{e}} L_{\text {radio }} B^{-3 / 2} / V$ (e.g., Govoni \& Fertti 2005), where $B$ is a magnetic flux density, $V \equiv \frac{4}{3} \pi r_{\text {cav }}^{3}$ is the volume of the cavity, and $C_{\mathrm{e}}$ is a constant depending on the spectral index $\alpha$ of the radio emission, as $C_{\mathrm{e}}=\sqrt{27 m_{\mathrm{e}}^{5} c^{9} /\left(2 \pi e^{7}\right)}\left(\nu_{1}^{-1 / 2}-\nu_{2}^{-1 / 2}\right) /\left(\log \nu_{2}-\log \nu_{1}\right)=1.4 \times 10^{8}[\operatorname{cgs}]$ for $\alpha=1, \nu_{1}=10 \mathrm{MHz}$, and $\nu_{2}=5 \mathrm{GHz}$. Therefore,

$$
\begin{aligned}
& \mathcal{K} P_{\mathrm{e}}=3.3 \times \\
&\left(\frac{\mathcal{K}}{100}\right)\left(\frac{L_{\text {radio }}}{9 \times 10^{37} \mathrm{cgs}}\right)\left(\frac{r_{\text {cav }}}{4 \mathrm{kpc}}\right)^{-3}\left(\frac{B}{10 \mu \mathrm{G}}\right)^{-\frac{3}{2}} \mathrm{eV} \mathrm{cm}^{-3} .
\end{aligned}
$$

On the other hand, the magnetic pressure is

$$
\mathcal{F} P_{\mathrm{B}}=\mathcal{F} \frac{B^{2}}{8 \pi}=2.5\left(\frac{\mathcal{F}}{1}\right)\left(\frac{B}{10 \mu \mathrm{G}}\right)^{2} \mathrm{eV} \mathrm{cm}^{-3} .
$$

Therefore, the total non-thermal pressure, $P_{\mathrm{T}} \equiv \mathcal{K} P_{\mathrm{e}}+\mathcal{F} P_{\mathrm{B}}$, takes the minimum at a certain value of $B=B_{\text {eq }}$, the so-called equipartition condition, which is calculated as

$$
\begin{aligned}
& B_{\text {eq }}=11\left(\frac{\mathcal{K} / \mathcal{F}}{100}\right)^{\frac{2}{7}}\left(\frac{L_{\text {radio }}}{9 \times 10^{37} \mathrm{cgs}}\right)^{\frac{2}{7}}\left(\frac{r_{\text {cav }}}{4 \mathrm{kpc}}\right)^{-\frac{6}{7}} \mu \mathrm{G}, \\
& P_{\mathrm{T}, \text { eq }}=\frac{7}{4} P_{\text {e,eq }}=\frac{7}{3} P_{\mathrm{B}, \text { eq }}=5.8 \times \\
& \left(\frac{\mathcal{K} / \mathcal{F}}{100}\right)^{\frac{4}{7}}\left(\frac{L_{\text {radio }}}{9 \times 10^{37} \mathrm{cgs}}\right)^{\frac{4}{7}}\left(\frac{r_{\text {cav }}}{4 \mathrm{kpc}}\right)^{-\frac{12}{7}} \mathrm{eV} \mathrm{cm}^{-3} .
\end{aligned}
$$

Thus the derived pressure at the equipartition condition is less than half of the ICM gas pressure, while the required equipartition magnetic flux density is typical for radio lobes of radio-loud AGNs (Kataoka \& Stawarz 2005). They have also found that the equipartition condition is achieved at least for the radio lobes of 40 radio galaxies. The discrepancy between $P_{\mathrm{T}, \text { eq }}$ and $P_{\text {gas }}$ may be explained by the underestimation of $\mathcal{K} / \mathcal{F} \sim 100$ or $L_{\text {radio }} \sim 9 \times 10^{37}$ erg s$^{-1}$, 
or by non-equilibrium situation. To make a balance between them, $\mathcal{K} / \mathcal{F}=690$ is needed. This value is consistent with those obtained by D05 for active bubbles in other clusters. It is also plausible that the radio intensity is getting dimmer in time due to the synchrotron cooling of relativistic electrons. We also note that the $L_{\text {radio }}$ value used for the calculation is probably overestimated, because the observed radio intensity is likely to include emission from the core regions. Radio observation with higher angular resolution is desired.

\subsection{Time scales}

For discussion of cooling and non-equilibrium effects, comparison of several time scales is important. First of all, synchrotron electron $\left(\gamma_{\mathrm{e}} \gtrsim 10^{4}\right)$ must be long-lived since the cavity is not fueled now from the central AGN. The synchrotron cooling time is calculated as

$$
t_{\mathrm{sync}}=\frac{9 m_{\mathrm{e}}^{3} c^{5}}{4 e^{4} B^{2} \gamma_{\mathrm{e}}}=25\left(\frac{B}{10 \mu \mathrm{G}}\right)^{-2}\left(\frac{\gamma_{\mathrm{e}}}{10^{4}}\right)^{-1} \mathrm{Myr} .
$$

Since rims of the cavities have not been shock heated, the cavities are supposed to have expanded at a velocity less than the sound speed, $v_{\mathrm{s}}=\sqrt{\gamma k T /\left(\mu m_{\mathrm{p}}\right)}=425 \mathrm{~km} \mathrm{~s}^{-1}$, where we have taken $k T=0.7 \mathrm{keV}, \gamma=5 / 3$ and $\mu=0.62$. Therefore, age of the cavities must be longer than the expansion time of

$$
t_{\mathrm{s}}=\frac{r_{\mathrm{cav}}}{v_{\mathrm{s}}}=9.2\left(\frac{r_{\mathrm{cav}}}{4 \mathrm{kpc}}\right) \mathrm{Myr}
$$

which is shorter than $t_{\text {sync. }}$. If we assume $\mathcal{K} / \mathcal{F}=690, t_{\text {sync }}$ can be shortened by a factor of $(\mathcal{K} / \mathcal{F} / 100)^{-4 / 7}=0.33$, and becomes comparable to $t_{\mathrm{s}}$. The time scale for the cavities to collapse when internal pressure has disappeared is also considered to be about $t_{\mathrm{s}}$. Such hollow cavities are buoyant and rise up outwards, even when pressure balance between inside and outside of the cavities is conserved. Churazov et al. (2001) have given the terminal velocity as $v_{\mathrm{t}}=\sqrt{2 g V / S C}$, where $S \equiv \pi r_{\text {cav }}^{2}$ is the cross section of the bubble, $g \equiv G M_{<R_{\text {cav }}} / R_{\text {cav }}^{2}$ is the gravity at the bubble, and $C=0.75$ is the drag coefficient. The travel time of the cavities to the current position is estimated to be

$$
\begin{aligned}
& t_{\text {buoy }}=R_{\text {cav }} / v_{\mathrm{t}}=15 \times \\
& \left(\frac{R_{\mathrm{cav}}}{7.4 \mathrm{kpc}}\right)^{2}\left(\frac{r_{\mathrm{cav}}}{4 \mathrm{kpc}}\right)^{-1 / 2}\left(\frac{M_{<R_{\mathrm{cav}}}}{2 \times 10^{11} M_{\odot}}\right)^{-1 / 2} \mathrm{Myr},
\end{aligned}
$$

which is comparable to or somewhat longer than $t_{\text {sync }}=8 \mathrm{Myr}$ with $\mathcal{K} / \mathcal{F}=690$. The refill time of the cavity is also given by McNamara et al. (2000) as,

$$
\begin{aligned}
& t_{\text {refill }}=2 R_{\text {cav }} \sqrt{r_{\text {cav }} /\left(G M_{<R_{\text {cav }}}\right)}=31 \times \\
& \quad\left(\frac{R_{\text {cav }}}{7.4 \mathrm{kpc}}\right)\left(\frac{r_{\text {cav }}}{4 \mathrm{kpc}}\right)^{1 / 2}\left(\frac{M_{<R_{\text {cav }}}}{2 \times 10^{11} M_{\odot}}\right)^{-1 / 2} \mathrm{Myr} .
\end{aligned}
$$

These considerations indicate that the cavity age of $t_{\mathrm{s}}$ or $t_{\text {buoy }} \sim 10 \mathrm{Myr}$ is much shorter than the group age of $\sim$ Gyr. Our time scales are consistent with B04, but in which they use $R_{\text {cav }}$ 
instead of $r_{\text {cav }}$.

\subsection{Energetics}

On the assumption that past AGN activity has produced the two cavities in about $\left(t_{\text {buoy }} / 2\right)$, we can estimate the required mechanical (kinetic) power of the AGN jets. A work to generate two cavities, $W_{\text {mech }} \equiv 2 P_{\text {gas }} V$, divided by $\left(t_{\text {buoy }} / 2\right)$ is called the mechanical luminosity (B04), and calculated to be

$$
\begin{aligned}
& L_{\text {mech }}=4 P_{\text {gas }} V / t_{\text {buoy }}=1.8 \times 10^{42} \times \\
& \left(\frac{R_{\text {cav }}}{7.4 \mathrm{kpc}}\right)^{-2}\left(\frac{r_{\text {cav }}}{4 \mathrm{kpc}}\right)^{\frac{7}{2}}\left(\frac{M_{<R_{\text {cav }}}}{2 \times 10^{11} M_{\odot}}\right)^{\frac{1}{2}} \operatorname{erg~s}^{-1} .
\end{aligned}
$$

It is supposed that this level of the AGN activity must continue for about $-t_{\text {buoy }}<t<-0.5 t_{\text {buoy }}$, and that it became inactive since $-0.5 t_{\text {buoy }}<t$. This value is comparable to the X-ray emission typically observed for LLAGN, although our upper limit on the X-ray luminosity is much lower, $L_{\mathrm{X}} \lesssim 10^{39} \mathrm{erg} \mathrm{s}^{-1}(0.5-4 \mathrm{keV})$. The observed radio emission around HCG $62 \mathrm{a}, L_{\text {radio }}=$ $1.8 \times 10^{38} \mathrm{erg} \mathrm{cm}^{-1}$, is also much lower than $L_{\text {mech }}$, as pointed out by B04.

Because there are no evidence for the strong AGN activity at present time, the central AGN, if exists, should have made a final outburst of total energy $\sim 10^{57}$ erg within $\sim 20$ Myr ago. Clearly, a single supernovae cannot account for this size of energy. Such an absence of strong X-ray or radio emission at the core is also noticed in NGC $4636\left(L_{\text {radio }}=1.4 \times 10^{38} \mathrm{erg} \mathrm{s}^{-1}\right.$; $L_{\mathrm{X}}<2.7 \times 10^{38} \mathrm{erg} \mathrm{s}^{-1}$; Ohto at al. 2003; O'Sullivan et al. 2005), which shows a disturbed Xray halo containing cavities associated with small-size jets. This might suggest that the AGN activity continues only for a short time scale, which is difficult to understand within the popular paradigm of AGN with a steady accretion disk. Formation of ghost cavities with an impulsively episodic activity of $\ll 10^{5} \mathrm{yr}$ is discussed by Wang \& Hu (2005). Considering captures of red giant stars by a super massive black hole $\left(M_{\mathrm{BH}}>2 \times 10^{8} M_{\odot}\right)$, its feedback energy can amount to $2.4 \times 10^{52}$ erg with a frequency of a few $10^{-5} \mathrm{yr}^{-1}$, which is too small to supply sufficient energy for the formation of cavities. It is probably true that HCG $62 \mathrm{a}$ also contains a massive black hole of $M_{\mathrm{BH}} \sim 10^{8} M_{\odot}$, because observations of the centers of nearby early-type galaxies show almost all have massive black hole (Tremaine et al. 2002). However, we have to consider switching (on $\rightarrow$ off) of the AGN activity.

\subsection{Difficulties in non-thermal pressure support by jets}

In $\S 8.2-8.4$, we have examined the properties of the cavities and the central AGN from the point of view that the non-thermal pressure of relativistic particles were provided through symmetrical jets of the past AGN activity. However there are several difficulties in this scenario.

As seen in $\S 8.2, \mathcal{K} / \mathcal{F}=690$ is needed to balance the ambient gas pressure with the internal non-thermal pressure at the equipartition condition. This value is in proportion to the inverse of $L_{\text {radio }}$, which is probably overestimated because significant fraction of $L_{\text {radio }}$ should be 
attributed to the core region instead of the radio robes (figure $3(\mathrm{a})$ ). Then $\mathcal{K} / \mathcal{F}=690$ becomes still larger, which is probably unrealistic. One possibility is that the relativistic electrons are fading due to the synchrotron loss, which may be justified by the fact that $t_{\text {sync }}$ under the equipartition magnetic field of $B_{\text {eq }}=17 \mu \mathrm{G}(\mathcal{K} / \mathcal{F}=690)$ is comparable to the estimated cavity age of $t_{\text {buoy }}$. The relativistic electrons have much shorter synchrotron cooling time than that of protons, hence apparently large $\mathcal{K} / \mathcal{F}$ might have been attained. In this case, the cavities are starting to collapse and losing the internal pressure support.

It is notable that both the two cavities observed in HCG 62 are pretty circular when projected in the sky (figure $3(\mathrm{a})$ ). The relative deviation image of figure 5 is remarkably smooth around the group core, and there are no obvious trails toward the cavities. This fact is quite difficult to understand considering the scenario that a pair of radio robes produced by symmetrical jets from the AGN has pushed away the IGM. In practice, clusters or galaxies hosting cavities usually show irregular or filamentary structures around the cluster core and cavities. The most prominent example is those of M 87 (Churazov et al. 2001; Young et al. 2002), in which the "trails" of the rising two radio bubbles are clearly seen in the X-ray image. Furthermore, our hollow sphere analysis suggests that the shape of cavities is probably elongated in the direction of our line of sight. It appears to be difficult for the symmetrical jets to make an elongation in such direction. We also note that shock heating is unlikely to be the origin of the cavities, because there is no significant evidence of heating at the edge of the cavities.

These considerations indicate that there might exist another supporting mechanism and/or formation scenario of the X-ray cavities, at least for HCG 62. In the following subsections, we consider these possibilities.

\subsection{Another supporting mechanism - hot gas clump -}

One possible supporting mechanism is a clump of hotter gas than the surrounding ICM $(k T=0.7 \mathrm{keV})$. For example, if we assume that the temperatures in the cavities are higher than that of ICM by three times, namely $T_{\mathrm{hc}}=3 T$, the required density, $n_{\mathrm{e}, \mathrm{hc}}$, is three times smaller. The emitted X-ray is roughly in proportion to $n_{\mathrm{e}, \mathrm{hc}}^{2} \sqrt{T_{\mathrm{hc}}}=0.2 n_{\mathrm{e}}^{2} \sqrt{T}$, hence the observed X-ray intensity can be as low as $20 \%$. Since we could have detected such hot emission if its flux were $\sim 20 \%$ of the $F_{\text {sphere }}$ in table 5 , we can derive the lower limit of the hot clump temperature, as $T_{\mathrm{hc}} \gtrsim 3 T$.

Schmidt et al. (2002) have done a similar discussion and ruled out volume-filling X-ray gas with temperature below $11 \mathrm{keV}$ for a cavity in the Perseus cluster. With regard to the origin of the hot gas clump, it is suggested that the jets may intrinsically contain protons, or that they may have captured ambient thermal protons possibly shock-heated in the very initial phase of the cavity formation (D05). If we observe in higher energy band, such hot emission can be detected as a hard tail of the spectrum. The hard $\Gamma=1.5$ power-law component observed 
with ASCA (Fukazawa et al. 2001) might originate from this kind of hot thermal emission, although the detected sky area of the power-law component is much more extended than the cavities.

If such a hot clump really exists, it undergoes a cooling by thermal conduction. Assuming $k T_{\mathrm{hc}}=3 k T \simeq 2 \mathrm{keV}$ and $n_{\mathrm{e}, \mathrm{hc}}=n_{\mathrm{e}} / 3 \simeq 10^{-3} \mathrm{~cm}^{-3}$, the thermal conductivity for a non-magnetized plasma is given by Spitzer (1962) as,

$$
\kappa_{\mathrm{S}}=10^{30}\left(\frac{k T_{\mathrm{hc}}}{2 \mathrm{keV}}\right)^{\frac{5}{2}}\left(\frac{n_{\mathrm{e}, \mathrm{hc}}}{10^{-3} \mathrm{~cm}^{-3}}\right)^{-1}\left(\frac{\ln \Lambda}{36}\right)^{-1} \mathrm{~cm}^{2} \mathrm{~s}^{-1},
$$

where $\ln \Lambda$ is the Coulomb logarithm. Therefore, the cooling time of the cavities is roughly calculated to be

$$
\begin{aligned}
& t_{\mathrm{cond}} \simeq \frac{r_{\mathrm{cav}}^{2}}{\kappa_{\mathrm{S}}}=0.5 \times \\
& \left(\frac{r_{\mathrm{cav}}}{4 \mathrm{kpc}}\right)^{2}\left(\frac{k T_{\mathrm{hc}}}{2 \mathrm{keV}}\right)^{-\frac{5}{2}}\left(\frac{n_{\mathrm{e}, \mathrm{hc}}}{10^{-3} \mathrm{~cm}^{-3}}\right) \mathrm{Myr},
\end{aligned}
$$

which is much shorter than the estimated cavity life span of $t_{\text {buoy }}$.

It is claimed that the thermal conductivity may become 5-10 times smaller than the Spitzer value under the turbulent magnetic fields (e.g., Chandran \& Maron 2004), although $t_{\text {cond }}$ seems to be still smaller considering this effect. On the other hand, from the observational point of view, we do see this kind of temperature variations in clusters. The most prominent example is the "cold front", first reported by Markevitch et al. (2000) for A2142. Ettori \& Fabian (2000) have pointed out that it requires the classical Spitzer thermal conductivity to be reduced at least by a factor of 250-2500. Markevitch et al. (2003) also find that $\sim 40$ times reduction is needed for A754. For the magnetic field expected in the radio robe $(B \sim 10 \mu \mathrm{G})$, the electron and proton gyro radii are by 11-12 orders of magnitude smaller than their Coulomb mean free paths, therefore the effective conductivity strongly depends on the topology of the field. If the magnetic field encloses the hot clump like a cage, the hot clump may survive for more than $\sim 20$ Myr.

\subsection{Another formation scenario — galaxy motion -}

As we found in $\S 3.3$ with the 2 -dimensional 2 - $\beta$ model fit, the location of HCG $62 \mathrm{a}$ (narrower component) is slightly offset from the group core (wider component) by about $5^{\prime \prime}$ $(1.5 \mathrm{kpc})$ on the projected sky image. This fact inevitably leads to the idea that the HCG $62 \mathrm{a}$ galaxy is moving around the group core. It is natural to consider that HCG $62 \mathrm{a}$ is performing a pseudo-Kepler motion, gradually decreasing its distance to the group core by a dragging force. From this point of view, the two cavities might be a piece of the trail of the HCG 62a orbit. Here, we assume that the orbital plane of HCG $62 \mathrm{a}$ is nearly in parallel to our line of sight, with a circular orbit. and that its orbital radius is close to the distance, $R_{\text {cav }}$, of the two cavities to the group core. 
It is interesting that the two cavities are located almost at the same distance from the group core, whereas the south cavity is by 1.6 times closer to HCG 62a than the north cavity. As indicated by a cross in figure 3 (a), HCG 62a is shifted from the group core indicated by a plus mark (center of the wider component) roughly toward the direction of the south cavity. These two facts support the assumption above. The measured redshift of HCG 62a is consistent with that of the group (table 1). On this assumption, the rotation speed is calculated to be

$v_{\text {rot }}=\sqrt{G M_{<\mathrm{R}_{\text {cav }}} / R_{\text {cav }}}=340 \mathrm{~km} \mathrm{~s}^{-1}$, which is comparable to the sound speed, $v_{\mathrm{s}}=425 \mathrm{~km} \mathrm{~s}^{-1}$. The period of rotation is

$$
t_{\mathrm{rot}}=\frac{2 \pi R_{\mathrm{cav}}}{v_{\mathrm{rot}}}=133\left(\frac{R_{\mathrm{cav}}}{7.4 \mathrm{kpc}}\right)^{\frac{3}{2}}\left(\frac{M_{<R_{\mathrm{cav}}}}{2 \times 10^{11} M_{\odot}}\right)^{-\frac{1}{2}} \mathrm{Myr} .
$$

Even though it is at least shorter than the group age, is much longer than other time scales, and the "tunnel" seems to be filled relatively quickly. In this scenario, the ICM gas would have experienced the encounter with the HCG 62a galaxy several times periodically. If some processes, e.g., freezing the plasma with magnetic field or the pressure support with hotter gas, have slowed the collapse of the cavities, this effect may be worthwhile consideration. In terms of the energetics, the kinetic energy of the galaxy motion amounts to $\sim 10^{59} \mathrm{erg}$, therefore it can supply sufficient energy to produce the cavities by depositing $7 \%$ of the kinetic energy per orbit. Such a motion of the galaxy also would have played an important role on the mixing and the metal enrichment of the ICM, which has been discussed in the previous section.

\section{Conclusion}

- We have carried out a detailed study on the hot-gas properties of the group of galaxies HCG 62, based on the data from Chandra and XMM-Newton. We confirmed the two cavities located almost symmetrically around the central galaxy.

- The size of spherical hollow cavities are constrained from the surface brightness structure to be $12^{\prime \prime}-17^{\prime \prime}$. The agreement with the observed angular size suggests that the gas density in the cavity is very low, less than $1 / 3$ and consistent with zero.

- The spectral fit indicated that the cavities were not caused by X-ray absorption. The observed temperature in the cavity region is consistent with that in the surrounding region.

- The spectrum within $4^{\prime}$ from the center requires two temperatures: $0.7 \mathrm{keV}$ and $1.4 \mathrm{keV}$. The cool component is centrally concentrated, narrower than the hot component, suggesting its association with the central galaxy HCG 62a.

- The mass profiles were obtained for the gas and stars. The hot component is much more extended than the stars, and thought to trace the gravitational potential of the galaxy group.

- The gravitational mass density drops steeply at about $5^{\prime}$ from the center. This is caused by the observed sharp drop of the temperature. There is a possibility that these regions are not in the hydrostatic equilibrium. 
- Abundance of $\mathrm{O}$ is $\sim 0.3$ solar, $\sim 3$ times less abundant than Fe and $\mathrm{Si}$, and shows a flatter profile. The shallow potential of HCG 62 is unable to confine the SN II products which should have been escaped in the form of galactic winds. The marginally higher $\mathrm{Mg} / \mathrm{O}$ ratio of $3.3 \pm 2.2$ implies steeper IMF.

- Abundances of Fe and Si show concentration in the central region, and a high $\mathrm{Ni} / \mathrm{Fe}$ ratio is suggested. These results are consistent with that they are synthesized by SN Ia in the central galaxy.

- The non-thermal energy density necessary to support the cavity implies $\mathcal{K} / \mathcal{F}=690$, namely almost 700 times larger energy than that of electrons needs to be contained the cavity. The lack of the central AGN or the trailing radio feature seems to suggests that the origin of the cavity in HCG 62 may not be directly related to AGN activities.

- We looked into alternative scenarios for the cavity creation. A clump of very hot gas and fast motion of the central galaxy were considered, but more observational evidences are necessary to perform a quantitative evaluation.

Thanks are given to an anonymous referee for useful comments which improved the original manuscript. Part of this work was financially supported by a Research Fellowship for Young Scientists from JSPS and Grant-in-Aid for Scientific Research (No. 16340077) from the Japan Society for the Promotion of Science, and also by a Grant-in-Aid of the Ministry of Education, Culture, Sports, Science and Technology (14079103; 16340077). N. O. acknowledges support from the Special Postdoctoral Researchers Program of RIKEN.

\section{References}

Anders, E., \& Grevesse, N. 1989, Geochim. Cosmochim. Acta, 53, 197

Bîizan, L., Rafferty, D. A., \& McNamara, B. R. 2004, ApJ, 607, 800

Boehringer, H., Voges, W., Fabian, A. C., Edge, A. C., \& Neumann, D. M. 1993, MNRAS, 264, L25

Brüggen, M., \& Kaiser, C. R. 2002, Nature, 418, 301

Buote, D. A. 2000a ApJ, 539, 172

Buote, D. A. 2000c MNRAS, 311, 176

Buote, D. A. 2002 ApJ, 574, L135

Buote, D. A., Lewis, A. D., Brighenti, F., \& Mathews, W. G. 2003a ApJ, 594, 741

Buote, D. A., Lewis, A. D., Brighenti, F., \& Mathews, W. G. 2003b ApJ, 595, 151

Chandran, B. D. G., \& Maron, J. L. 2004, ApJ, 602, 170

Churazov, E., Brüggen, M., Kaiser, C. R., Böhinger, H., \& Forman, W. 2001, ApJ, 554, 261

Condon, J. J., Cotton, W. D., Greisen, E. W., Yin, Q. F., Perley, R. A., Taylor, G. B., \& Broderick, J. J. 1998, AJ, 115, 1693

Coziol, R., Ribeiro, A. L. B., Carvalho, R. R., \& Capelato, H. V. 1998 ApJ, 493, 563

Coziol, R., Brinks, E., \& Bravo-Alfaro, H. 2004, AJ, 128, 68

De Grandi, S., Ettori, S., Longhetti, M., \& Molendi, S. 2004, A\&A, 419, 7 
Dunn, R. J. H., \& Fabian, A. C. 2004, MNRAS, 355, 862

Dunn, R. J. H., Fabian, A. C., \& Taylor, G. B. 2005, MNRAS, 364, 1343

Ettori, S., \& Fabian, A. C. 2000, MNRAS, 317, L57

Finoguenov, A., \& Ponman, T. J. 1999, MNRAS, 305, 325

Finoguenov, A., \& Jones, C. 2001, ApJL, 547, L107

Fabian, A. C. et al. 2000, MNRAS, 318, L65

Fabian, A. C., Celotti, A., Blundell, K. M., Kassim, N. E., \& Perley, R. A. 2002, MNRAS, 331, 369

Fukazawa, Y., Nakazawa, K., Isobe, N., Makishima, K., et al. 2001, ApJL, 546, 87

Govoni, F. \& Fertti, L. 2005 IJM Phys D,

Grant, C. E., Bautz, M. W., Kissel, S. M., LaMarr, B., \& Prigozhin, G. Y. 2005, Proc. SPIE, 5898, 201

Grevesse, N., \& Sauval, A. J. 1998, Space Science Reviews, 85, 161

Harrison, F. A., Eckart, M. E., Mao, P. H., Helfand, D. J., \& Stern, D. 2003, ApJ, 596, 944

Hickson, P., Kindl, E., \& Auman, J. R. 1989, ApJS, 70, 687

Ikebe, Y., Makishima, K., Fukazawa, Y., Tamura, T., Xu, H., Ohashi, T., \& Matsushita, K. 1999, ApJ, 525, 58

Inoue, H., Koyama, K., Matsuoka, M., Ohashi, T., Tanaka, Y., \& Tsunemi, H. 1980, ApJ, 238, 886

Iwamoto, K., Brachwitz, F., Nomoto, K., Kishimoto, N., Umeda, H., Hix, W. R., \& Thielemann,

F.-K. 1999, ApJS, 125, 439

Kataoka, J., \& Stawarz, L. 2005 ApJ, 622, 797

Katayama, H., Takahashi, I., Ikebe, Y., Matsushita, K., \& Freyberg, M. J. 2004, A\&A, 414, 767

LaMarr, B., 2000, ACIS Memo \#181, "Resolution of ACIS Devices at -110C, -120C, -123C", http://cxc.harvard.edu/ cal/Acis/Cal_prods/eres/in_flight/plotNewW123/ plotNewW123.ps

Markevitch, M. http://cxc.harvard.edu/contrib/maxim/acisbg/

Markevitch, M., et al. 2000, ApJ, 541, 542

Matsushita, K., Belsole, E., Finoguenov, A., Boehringer, H. 2002, A\&A, 386, 77

Matsushita, K., Finoguenov, A. \& Böhringer., H. 2003, A\&A, 401, 443

Markevitch, M., et al. 2003, ApJL, 586, L19

McNamara, B. R., et al. 2000, ApJL, 534, L135

McNamara, B. R., et al. 2001, ApJL, 562, L149

Mulchaey, J. S., \& Zabludoff, A. I. 1998, ApJ, 496, 73

Mulchaey, J. S., Davis, D. S., Mushotzky, R. F., \& Burstein, D. 2003, ApJS, 145, 39

Mushotzky, R., Figueroa-Feliciano, E., Loewenstein, M., \& Snowden, S. L. 2003 [arXiv:astro$\mathrm{ph} / 0302267]$

Mazzotta, P., Kaastra, J. S., Paerels, F. B., Ferrigno, C., Colafrancesco, S., Mewe, R., \& Forman, W. R. 2002, ApJL, 567, L37

Nevalainen, J., Markevitch, M., \& Lumb, D. 2005, ApJ, 629, 172

Nomoto, K., Tominaga, N., Umeda, H., Kobayashi, C., \& Maeda, K. 2006, [arXiv:astro-ph/0605725]

Ohto, A., Kawano, N., \& Fukazawa, Y. 2003 PASJ, 55, 819

O’Sullivan, E., Vrtilek, J. M., \& Kempner, J. C. 2005, ApJ, 624, L77

Pildis, R. A., Bregman, J. N., \& Evrard, A. E. 1995, ApJ, 443, 514 
Ponman, T. J., \& Bertram. 1993, Nature, 363, 6

Read, A. M., \& Ponman, T. J. 2003, A\&A, 409, 395

Schmidt, R. W., Fabian, A. C., \& Sanders, J. S. 2002, MNRAS, 337, 71

Shimada, M., Ohyama, Y., Nishiura, S., Murayama, T., \& Taniguchi, Y. 2000, AJ, 119, 2664

Spitzer, L. 1962, Physics of Fully Ionized Gases (2nd ed.; New York: Wiley)

Stevens, J. B., Webster, R. L., Barnes, D. G., Pisano, D. J., \& Drinkwater, M. J. 2004, Publications of the Astronomical Society of Australia, 21, 318

Tamura, T., Kaastra, J. S., Makishima, K., \& Takahashi, I. 2003, A\&A, 399, 497

Thielemann, F.-K., Nomoto, K., \& Hashimoto, M. 1996, ApJ, 460, 408

Tran, K.-V. H., Simard, L., Zabludoff, A. I., \& Mulchaey, J. S. 2001, ApJ, 549, 172

Tremaine, S., et al. 2002, ApJ, 574, 740

Tsujimoto, T., Nomoto, K., Yoshii, Y., Hashimoto, M., Yanagida, S., \& Thielemann, F.-K. 1995, MNRAS, 277, 945

Verdes-Montenegro, L., Yun, M. S., Williams, B. A., Huchtmeier, W. K., Del Olmo, A., \& Perea, J. 2001, A\&A, 377, 812

Voit, G. M. 2005, Reviews of Modern Physics, 77, 207

Vrtilek et al. Dec, 08, 2001, Chandra News Releases http://chandra.harvard.edu/photo/2001/hcg62/

Vrtilek, J. M, Grego, L., David, L. P., Ponman, T. J., Forman, W., Jones, C., \& Harris, D. E. 2002, APS Meeting, B17.107,

Wang, J. M., \& Hu, C. 2005, ApJ, 630, L125

Xu, H., Kahn, S. M., Peterson, J. R. Behar, E. Paerels, F. B. S., Mushotzky, R. F. Jernigan, J. G. Brinkman, A. C. \& Makishima, K. 2002, ApJ, 579, 600

Xue, Y. J., Böhringer, H., \& Matsushita, K. 2004, A\&A, 833, 845

Young, P. J. 1976, AJ, 81, 807

Young, A. J., Wilson, A. S., \& Mundell, C. G. 2002, ApJ, 579, 560

Zabludoff, A. I., \& Mulchaey, J. S. 2000, ApJ, 539, 136 